\title{
Nonlinear compressive stability of hyperelastic 2D lattices at finite volume fractions
}

\author{
Bluhm, Gore Lukas; Sigmund, Ole; Wang, Fengwen; Poulios, Konstantinos
}

Published in:

Journal of the Mechanics and Physics of Solids

Link to article, DOI:

10.1016/j.jmps.2019.103851

Publication date:

2020

Document Version

Peer reviewed version

Link back to DTU Orbit

Citation $(A P A)$ :

Bluhm, G. L., Sigmund, O., Wang, F., \& Poulios, K. (2020). Nonlinear compressive stability of hyperelastic 2D lattices at finite volume fractions. Journal of the Mechanics and Physics of Solids, 137, [103851]. https://doi.org/10.1016/j.jmps.2019.103851

\section{General rights}

Copyright and moral rights for the publications made accessible in the public portal are retained by the authors and/or other copyright owners and it is a condition of accessing publications that users recognise and abide by the legal requirements associated with these rights.

- Users may download and print one copy of any publication from the public portal for the purpose of private study or research.

- You may not further distribute the material or use it for any profit-making activity or commercial gain

- You may freely distribute the URL identifying the publication in the public portal 


\title{
Nonlinear compressive stability of hyperelastic 2D lattices at finite volume fractions
}

\author{
Gore Lukas Bluhm*, Ole Sigmund, Fengwen Wang, Konstantinos Poulios \\ Department of Mechanical Engineering, Technical University of Denmark, Nils Koppels Allé, Building 404, 2800 Kgs. Lyngby, Denmark
}

\begin{abstract}
A framework is introduced for benchmarking periodic microstructures in terms of their ability to maintain their stiffness under large deformations, accounting in a unified manner both for buckling and softening due to geometric and material nonlinearities. The proposed framework is applied to three classical 2D lattice microstructures at different volume fractions as well as to an optimized hierarchical microstructure from the literature. The high slenderness of the structure members, often assumed in analyses, is demonstrated not to be valid at volume fractions of $10 \%$ and above, with the infinitesimal volume fraction solutions underestimating the actual buckling resistance considerably. The performed analyses provide useful and quantitative insight regarding the compressive load carrying capacity of materials with a moderately dense periodic microstructure, in a rather universal and practical form.
\end{abstract}

Keywords: buckling; anisotropic material; stability and bifurcation; finite strain; non-slender lattice structures

\section{Introduction}

Within structural and material mechanics, lattice structures have important applications in a wide range of length scales [1, 2, 3, 4] and have therefore been subject of extensive studies, both numerically and experimentally [5, 6, 7, 8]. Typically, numerical studies deal with the evaluation of lattices in terms of stiffness per volume fraction [9, 10, 11, 12], plastic yield or compressive instabilities [13, 14, 15, 16, 17, 18], elastic or plastic anisotropy [19, 20, 21], and effective fracture mechanical properties [22, 23, 24, 25].

Depending on the geometry of a given microstructure, models of varying complexity may be necessary for investigating its response to mechanical loading. For studying, for instance, the load carrying capacity of low volume fraction and geometrically simple microstructures, beam element models are often sufficiently accurate [26, 27, 19, 28]. Also depending on the kind of the study performed, geometrically linear models may be sufficient but often, geometrical nonlinearities are essential [27, 17]. Especially at larger volume fraction values, on one hand, beam elements fall short in representing the actual geometry [9]. On the other hand, maximum loads become large enough for finite strain effects in the microstructure to become essential [29].

Results from analytical or numerical eigenvalue buckling analysis and Bloch wave theory [14] have unquestionably been of immense value for estimating the load carrying capacity and understanding the behavior of lattice materials under compressive loads. Nevertheless, microstructures of increased geometrical complexity, combined with large macro-scale deformations and finite strain effects at the micro-scale, render eigenvalue buckling analysis difficult to apply and interpret. Oppositely, direct force or displacement controlled nonlinear continuum mechanics simulations are generic, relatively simple to apply, resemble the real system directly and are hence more straightforward to interpret. However, they require the application of appropriate perturbations, representative of the real system under consideration and boundary conditions must be imposed that cover both local and global modes. Direct fully nonlinear continuum mechanics simulations are used in the present work to cover hitherto scarcely treated effects in lattice structures, such as

- resolving the actual deformation fields in joints to avoid simplifications of joint stiffness,

- finite-strain effects such as beam thickening due to non-zero Poisson ratio [29],

- progressive softening of the structure without a sharp instability point,

- possibly evolving arbitrary misalignment between applied principal stress axes and lattice symmetry axes [30],

- accurate modeling of general hierarchical microstructures.

*glubluh@mek.dtu.dk 
Especially for elastomers, that can sustain large strains without undergoing plastic deformation, the aforementioned effects become highly relevant already for moderate material volume fractions.

The proposed generic approach with all geometrical and material nonlinear effects included, becomes increasingly relevant with recent achievements in manufacturing technology that allow for the design of materials with extremal physical properties such as stiffness [31] and buckling resistance [32]. It is also highly relevant in connection to the newer trend in lattice material research with focus on exploitation of buckling-like response, rather than considering buckling as a failure mode, for instance for energy absorption with recoverability [33] or for achieving radical transitions in material properties based on geometrical changes under loading [34].

One particular area of interest for the present work is anisotropy. As opposed to linearized elasticity, where the anisotropy of any lattice structure is described by a finite number of elasticity constants according to the lattice's Lau group [35], there is in general no simple description for the anisotropically nonlinear response of a lattice structure. For defining yield surfaces for lattice structures, for instance, one approach is to provide certain 2D or 3D slices of higher dimensional normal and shear stress spaces [36, 19], while another approach is to investigate the response only to uniaxial loading in any orientation [21]. The often implicit convention of reporting anisotropic yield surfaces in principal stress space, at a fixed orientation with respect to the lattice axes, actually covers only a small fraction of the anisotropic behaviour of lattice materials.

The following section introduces the periodic cell model used in the subsequent studies. Special focus is placed on the periodic boundary conditions implemented in the model that allow for a generalised application of a macroscale stress or strain field under large deformations. Other modelling aspects, such as the chosen load cases and the definition of perturbations are discussed in Section 2, as well. Section 3 explains the interpretation of the obtained results and their presentation in the subsequent Section 4 , in dimensionless form. The provided results cover the evaluation of the nonlinear response of square, triangular and hexagonal lattices at three volume fractions up to $50 \%$ and comparison with a hierarchical lattice at $30 \%$ volume fraction from the literature, optimized for buckling resistance. The most important findings are summarized in the concluding Section 5.

\section{Numerical method}

Finite strain continuum mechanics provide a sufficiently generic framework for benchmarking periodic microstructures of arbitrary geometrical complexity and volume fraction in terms of their nonlinear static response. A periodic cell model is hence used in the present study, based on a finite element discretization of a plane strain hyperelastic continuum. Such a continuum representation allows to study in a unified manner both simple and complex geometries at both low and high volume fraction levels.

Among all available hyperelastic laws, the specific choice of a finite strain elastic constitutive law at the continuum level, might theoretically affect the observed response at the microstructure level. For moderate strains though, the differences between many hyperelastic laws are relatively small. For simplicity, the well established compressible neo-Hookean material according to [37] is used to describe the constitutive behavior at the continuum level. The chosen material law results in a relation between the deformation gradient $\tilde{\boldsymbol{F}}$ and Kirchhoff stress $\tilde{\boldsymbol{\tau}}$ given as

$$
\tilde{\boldsymbol{\tau}}=K \ln |\tilde{\boldsymbol{F}}| \boldsymbol{I}+G|\tilde{\boldsymbol{F}}|^{-2 / 3} \operatorname{dev}\left(\tilde{\boldsymbol{F}} \tilde{\boldsymbol{F}}^{T}\right)
$$

with $K$ and $G$ being the initial bulk and shear modulus of the material, respectively. Since all results are presented in dimensionless form, they are independent of the actual scaling of the material stiffness. However, it is important to report the adopted initial Poisson ratio $v=0.4$, corresponding to $K / G=14: 3$, used in the majority of the simulations, unless otherwise stated. The choice of a relatively high value is justified as representative for polymeric materials.

A plane strain finite element model for the periodic cell is implemented in the GetFEM [38] high-level finite element framework, which allows for an easy coupling between the discretized displacement field unknown and scalar homogenized strain unknowns, presented below. Quadratic isoparametric 9-node quadrilateral and 6-node triangular elements are used for representing the geometry and the displacements field on unstructured meshes. Apart from the otherwise standard numerical framework, a new implementation was necessary for applying a generic loading under large deformations in combination with the enforcement of periodicity conditions and this is explained in detail below. Moreover, the load cases adopted in this study and the types of imperfections imposed are introduced and justified as essential elements of the numerical model. 


\subsection{Representative periodic cell model}

Compared to the small deformations case, applying a stress state to the periodic cell of an anisotropic microstructure undergoing large deformations is not as trivial. The main difficulty is conceptually illustrated in Figure 1, where the same microstructure is loaded with the same Cauchy stress components in two cases resulting in different material axes rotations, depending on the far field boundary conditions.
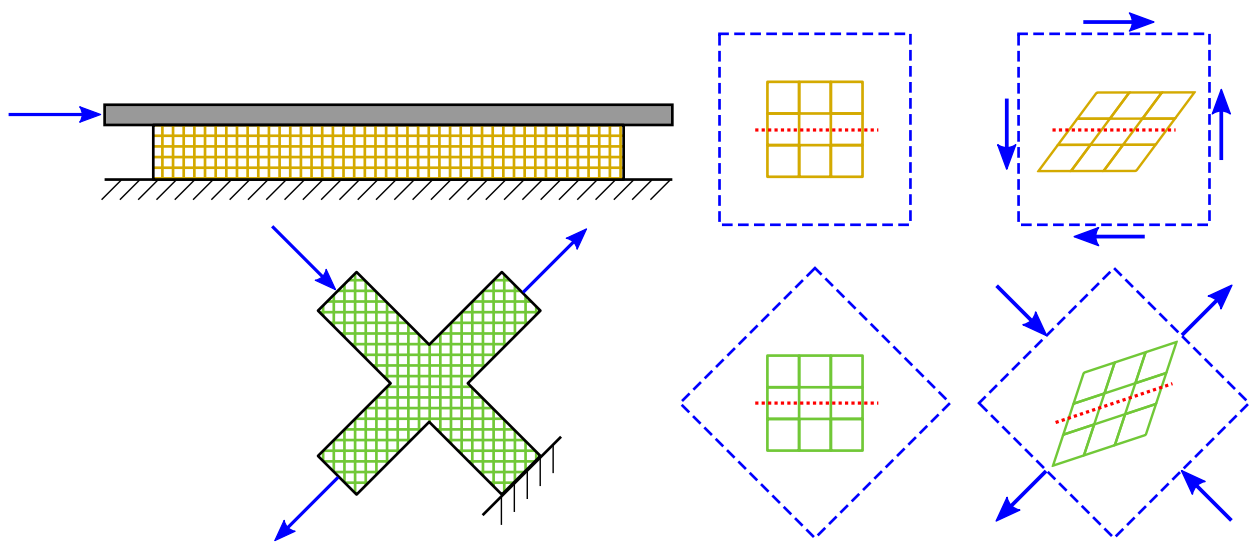

Figure 1: Two cases with the same initial material orientation and under the same stress state but with differently evolving material axes due to different boundary conditions.

Especially in the context of stability investigations, it is absolutely essential to explicitly choose and state how the load frame will evolve compared to the material frame during loading. It is possible for example to define the homogenized stress state in a frame that follows a given axis in the material microstructure, whether it is a symmetry or an arbitrary axis.

The periodic cell model employed in this work is defined and numerically implemented in the $X Y$ frame shown in Figure 2, which is typically aligned with some symmetry axis in the material microstructure. However, in order to achieve a fine control over the relative rotations between the applied load and deformations, both initially and during loading, the homogenized deformation and stress states acting on the periodic cell are defined in a different frame $x y$.

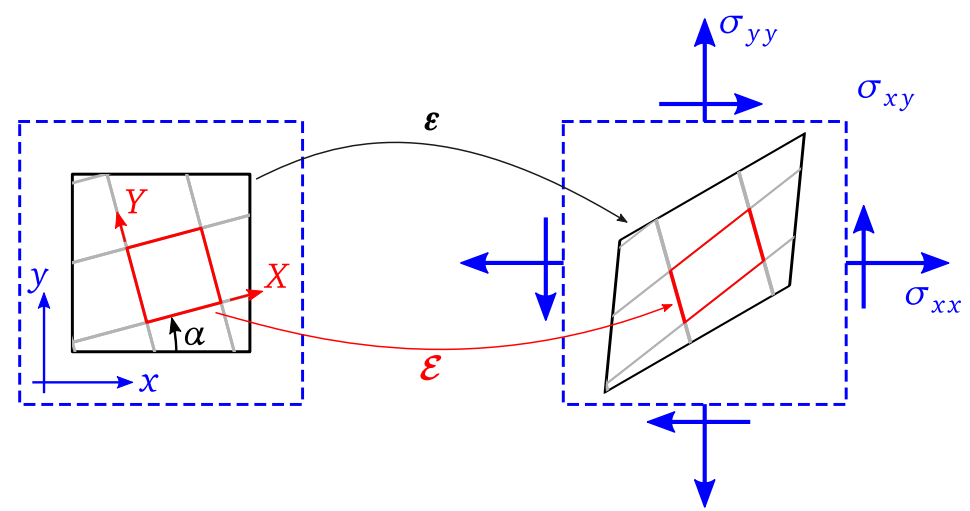

Figure 2: Embedding of the periodic cell represented in the $X Y$ frame in a lattice material under homogeneous deformation defined in the $x y$ frame. The angle $\alpha$ specifies the relative rotation between the two frames that are both defined in the undeformed configuration.

The overall deformation and rotation of the periodic cell is defined by the four components of the macroscopic strain tensor defined in the $x y$ frame

$$
\boldsymbol{\varepsilon}=\left[\begin{array}{ll}
\partial u_{x} / \partial x & \partial u_{x} / \partial y \\
\partial u_{y} / \partial x & \partial u_{y} / \partial y
\end{array}\right]=\left[\begin{array}{ll}
\varepsilon_{x x} & \varepsilon_{x y} \\
\varepsilon_{y x} & \varepsilon_{y y}
\end{array}\right]
$$


where $\boldsymbol{u}=\left(u_{x}, u_{y}\right)$ is an assumed homogenized displacement field of the continuum that the cell is embedded in. For the periodic cell problem defined here, the four components of the tensor $\varepsilon$ are assumed as independent problem variables.

For a rotation angle $\alpha$ from the $x y$ to the $X Y$ frame, the corresponding strain components in the $X Y$ frame are given by the known transformation

$$
\left[\begin{array}{ll}
\mathcal{E}_{X X} & \mathcal{E}_{X Y} \\
\mathcal{E}_{Y X} & \mathcal{E}_{Y Y}
\end{array}\right]=\left[\begin{array}{cc}
c & s \\
-s & c
\end{array}\right]\left[\begin{array}{ll}
\varepsilon_{x x} & \varepsilon_{x y} \\
\varepsilon_{y x} & \varepsilon_{y y}
\end{array}\right]\left[\begin{array}{cc}
c & -s \\
s & c
\end{array}\right],
$$

as linear functions of the four aforestated independent strain variables, with $c=\cos \alpha$ and $s=\sin \alpha$.

After imposing a certain deformation state on the periodic cell through a given strain tensor $\varepsilon$, it is straightforward to numerically solve the microscale boundary value problem and obtain the homogenized first Piola-Kirchhoff stress components $P_{X X}, P_{X Y}, P_{Y X}$ and $P_{Y Y}$, acting on the cell. They correspond to resultant force components on the sides of the periodic cell divided by the corresponding areas in the undeformed state of the cell. These stress components can then be further converted to Cauchy stresses in the global frame $x y$ according to the transformation

$$
\left[\begin{array}{ll}
\sigma_{x x} & \sigma_{x y} \\
\sigma_{x y} & \sigma_{y y}
\end{array}\right]=\frac{1}{|\boldsymbol{F}|}\left[\begin{array}{cc}
c & -s \\
s & c
\end{array}\right]\left[\begin{array}{cc}
P_{X X} & P_{X Y} \\
P_{Y X} & P_{Y Y}
\end{array}\right]\left[\begin{array}{cc}
c & s \\
-s & c
\end{array}\right] \boldsymbol{F}^{T}, \quad \text { with } \boldsymbol{F}=\boldsymbol{I}+\boldsymbol{\varepsilon},
$$

which is linear with respect to the input stresses but nonlinear with respect to the components of $\varepsilon$. This expression for the Cauchy stress tensor can be used for post-processing purposes or for applying certain constraints on some of its components, as will be shown below.

So far introduced strain and stress quantities define, in the $x y$ frame, the assumed homogeneous deformation and stress state of the lattice material, that the considered periodic cell is part of. These act as boundary conditions for the continuum mechanical periodic cell model illustrated in Figure 3 , with the undeformed solid domain $\Omega$ and boundaries $\Gamma_{L}, \Gamma_{B}, \Gamma_{R}, \Gamma_{T}$ at the left, bottom, right and top, respectively. Since the microstructure is assumed periodic, a one to one mapping between left and right as well as bottom and top boundaries of the periodic cell is postulated.

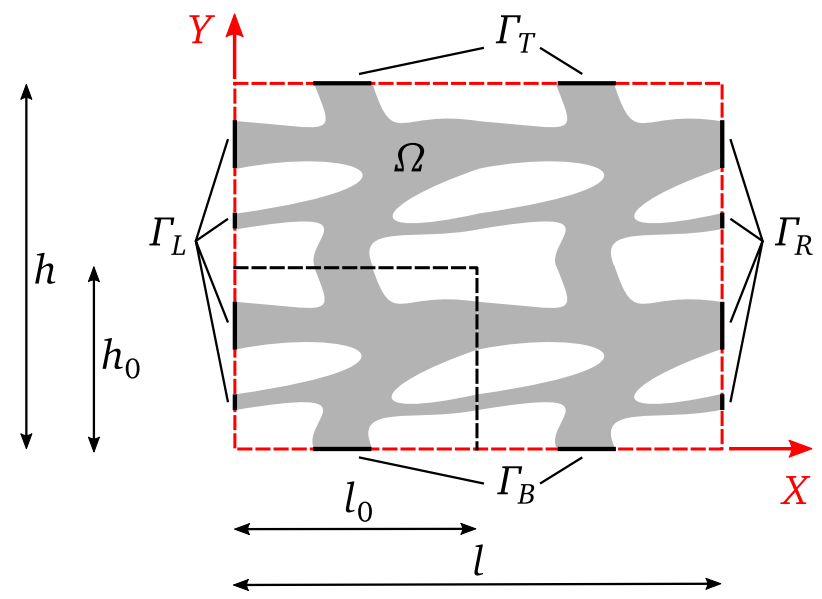

Figure 3: Continuum mechanical periodic cell model of dimensions $h \times l$, which can include several unit-cells of dimensions $h_{0} \times l_{0}$, as for example the here illustrated $2 \times 2$ unit-cells. $\Omega$ is the solid domain and $\Gamma_{L}, \Gamma_{B}, \Gamma_{R}, \Gamma_{T}$ are its periodic boundaries

The deformation of the microstructure is represented through a displacement field $\tilde{\boldsymbol{u}}$, defined in $\Omega$, and periodicity constraints are imposed through vector valued Lagrange multiplier fields $\boldsymbol{\Lambda}_{R}$ and $\boldsymbol{\Lambda}_{T}$, defined on $\Gamma_{R}$ and $\Gamma_{T}$. Assuming no body forces, the balance of linear momentum within the periodic cell domain can then be expressed in weak form as

$$
\int_{\Omega} \tilde{\boldsymbol{\tau}}:\left(\nabla \boldsymbol{\delta} \tilde{\boldsymbol{u}} \tilde{\boldsymbol{F}}^{-1}\right) d V-\int_{\Gamma_{R}} \boldsymbol{\Lambda}_{R} \cdot\left(\boldsymbol{\delta} \tilde{\boldsymbol{u}}-\boldsymbol{\delta} \tilde{\boldsymbol{u}}_{R \rightarrow L}\right) d Y-\int_{\Gamma_{T}} \boldsymbol{\Lambda}_{T} \cdot\left(\boldsymbol{\delta} \tilde{\boldsymbol{u}}-\boldsymbol{\delta} \tilde{\boldsymbol{u}}_{T \rightarrow B}\right) d X=0,
$$

where $\tilde{\boldsymbol{\tau}}$ is given by Eq. (1) and $\tilde{\boldsymbol{F}}=\boldsymbol{I}+\nabla \tilde{\boldsymbol{u}}$. Eq. (5) has to be fulfilled for any admissible variation $\delta \tilde{\boldsymbol{u}}$. The mappings $R \rightarrow L$ and $T \rightarrow B$ denote evaluation of the concerned quantity at a point of the opposite side $\Gamma_{L}$ or $\Gamma_{B}$, that corresponds to the current integration point on $\Gamma_{R}$ or $\Gamma_{T}$, respectively. 
The way the Lagrange multiplier fields are used in Eq. (1), acting directly on variations of $\tilde{\boldsymbol{u}}$ in an integral defined in the undeformed configuration, implies that $\boldsymbol{\Lambda}_{R}$ and $\boldsymbol{\Lambda}_{T}$ are tractions in the sense of first Piola-Kirchhoff stresses. Consequently the averaging equations

$$
\left(\begin{array}{l}
P_{X X} \\
P_{Y X}
\end{array}\right)=\frac{1}{h} \int_{\Gamma_{R}} \Lambda_{R} d Y \quad \text { and } \quad\left(\begin{array}{l}
P_{X Y} \\
P_{Y Y}
\end{array}\right)=\frac{1}{l} \int_{\Gamma_{T}} \Lambda_{T} d X
$$

hold by definition of the first Piola-Kirchhoff stress tensor.

Periodicity conditions including the superposition of an average strain $\varepsilon$ can be expressed in weak form as

$$
\begin{aligned}
& \int_{\Gamma_{R}}\left(-\tilde{\boldsymbol{u}}+\tilde{\boldsymbol{u}}_{R \rightarrow L}+l\left[\begin{array}{cc}
c & s \\
-s & c
\end{array}\right]\left[\begin{array}{ll}
\varepsilon_{x x} & \varepsilon_{x y} \\
\varepsilon_{y x} & \varepsilon_{y y}
\end{array}\right]\left(\begin{array}{l}
c \\
s
\end{array}\right)\right) \cdot \boldsymbol{\delta} \boldsymbol{\Lambda}_{R} d Y \\
+ & \int_{\Gamma_{T}}\left(-\tilde{\boldsymbol{u}}+\tilde{\boldsymbol{u}}_{T \rightarrow B}+h\left[\begin{array}{cc}
c & s \\
-s & c
\end{array}\right]\left[\begin{array}{cc}
\varepsilon_{x x} & \varepsilon_{x y} \\
\varepsilon_{y x} & \varepsilon_{y y}
\end{array}\right]\left(\begin{array}{c}
-s \\
c
\end{array}\right)\right) \cdot \boldsymbol{\delta} \boldsymbol{\Lambda}_{T} d X=0,
\end{aligned}
$$

that has to hold for any admissible variation $\delta \boldsymbol{\Lambda}_{R}$ or $\delta \boldsymbol{\Lambda}_{T}$ of the multipliers. Eq. (7) imposes the homogenized strains as a boundary condition for the microscale displacements field by coupling the scalar variables $\boldsymbol{\varepsilon}$ and the field variables $\tilde{\boldsymbol{u}}$, $\boldsymbol{\Lambda}_{R}$ and $\boldsymbol{\Lambda}_{T}$. This condition has to be complemented with the overall force equilibrium for the periodic cell, expressed in weak form through variations of the independent strain variables $\varepsilon_{x x}, \varepsilon_{x y}, \varepsilon_{y x}$ and $\varepsilon_{y y}$. The weak form equation

$$
\begin{aligned}
& \int_{\Gamma_{R}}\left(l\left[\begin{array}{cc}
c & s \\
-s & c
\end{array}\right]\left[\begin{array}{ll}
\delta \varepsilon_{x x} & \delta \varepsilon_{x y} \\
\delta \varepsilon_{y x} & \delta \varepsilon_{y y}
\end{array}\right]\left(\begin{array}{l}
c \\
s
\end{array}\right)\right) \cdot \Lambda_{R} d Y \\
+ & \int_{\Gamma_{T}}\left(h\left[\begin{array}{cc}
c & s \\
-s & c
\end{array}\right]\left[\begin{array}{ll}
\delta \varepsilon_{x x} & \delta \varepsilon_{x y} \\
\delta \varepsilon_{y x} & \delta \varepsilon_{y y}
\end{array}\right]\left(\begin{array}{c}
-s \\
c
\end{array}\right)\right) \cdot \Lambda_{T} d X-\operatorname{lh}\left[\begin{array}{cc}
\delta \varepsilon_{x x} & \delta \varepsilon_{x y} \\
\delta \varepsilon_{y x} & \delta \varepsilon_{y y}
\end{array}\right]:\left[\begin{array}{ll}
P_{x x} & P_{x y} \\
P_{y x} & P_{y y}
\end{array}\right]=0 .
\end{aligned}
$$

has to hold for any of the four variations $\delta \varepsilon_{x x}, \delta \varepsilon_{x y}, \delta \varepsilon_{y x}$ and $\delta \varepsilon_{y y}$. The first Piola-Kirchhoff stress components in the $x y$ coordinate system, appearing in Eq. (8), are considered as given quantities if the corresponding strains are unknown.

The four scalar equations contained in Eq. (8) allow in principle to prescribe any combination of four strain or stress components. For those strain components in $\varepsilon$ which are prescribed, the corresponding variations in Eq. (8) are disregarded and these components are simply treated as known quantities instead of problem variables. For those first Piola-Kirchhoff stress components that are prescribed, typically set equal to zero, the prescribed value is accounted for in Eq. (8).

Another special case of interest is for a fixed ratio between two components of the Cauchy stress tensor in the $x y$ frame. If the ratio $\sigma_{x x} / \sigma_{y y}=k$ is known, for instance, the corresponding condition can, based on Eqs. (4) and (6), be expressed in terms of the Lagrange multiplier fields $\boldsymbol{\Lambda}_{R}$ and $\boldsymbol{\Lambda}_{T}$, as

$$
k=\frac{\left(\frac{c+c \varepsilon_{x x}+s \varepsilon_{x y}}{h} \int_{\Gamma_{R}} \Lambda_{R} d Y-\frac{s+s \varepsilon_{x x}-c \varepsilon_{x y}}{l} \int_{\Gamma_{T}} \Lambda_{T} d X\right) \cdot\left(\begin{array}{c}
c \\
-s
\end{array}\right)}{\left(\frac{s+s \varepsilon_{y y}+c \varepsilon_{y x}}{h} \int_{\Gamma_{R}} \Lambda_{R} d Y+\frac{c+c \varepsilon_{y y}-s \varepsilon_{y x}}{l} \int_{\Gamma_{T}} \Lambda_{T} d X\right) \cdot\left(\begin{array}{l}
s \\
c
\end{array}\right)}
$$

which can be imposed through the following weak form expression

$$
\begin{aligned}
& l \int_{\Gamma_{R}}\left[\begin{array}{ll}
c & s
\end{array}\right]\left[\begin{array}{cc}
1+\varepsilon_{x x} & \varepsilon_{y x} \\
\varepsilon_{x y} & 1+\varepsilon_{y y}
\end{array}\right]\left[\begin{array}{cc}
c & -s \\
-k s & -k c
\end{array}\right] \boldsymbol{\Lambda}_{R} \delta \varepsilon_{x x} d Y \\
& +h \int_{\Gamma_{T}}\left[\begin{array}{ll}
-s & c
\end{array}\right]\left[\begin{array}{cc}
1+\varepsilon_{x x} & \varepsilon_{y x} \\
\varepsilon_{x y} & 1+\varepsilon_{y y}
\end{array}\right]\left[\begin{array}{cc}
c & -s \\
-k s & -k c
\end{array}\right] \boldsymbol{\Lambda}_{T} \delta \varepsilon_{x x} d X=0
\end{aligned}
$$

that has to hold for any variation $\delta \varepsilon_{x x}$, instead of the corresponding equation in Eq. (8).

So far the periodic cell problem is defined in terms of the three unknown vector fields $\tilde{\boldsymbol{u}}, \boldsymbol{\Lambda}_{R}$ and $\boldsymbol{\Lambda}_{T}$ as well as the four scalar variables $\varepsilon_{x x}, \varepsilon_{x y}, \varepsilon_{y x}$ and $\varepsilon_{y y}$, and sufficient equations are provided for solving for all unknowns. Different kinds of loadings can be implemented by making specific choices about prescribed strain components and possibly replacing some of the four equations contained in Eq. (8) with alternative ones, like e.g. Eq. (10). Since rigid body rotation is already treated in the presented equations, the only remaining condition for the periodic cell problem to be complete, is to restrain rigid body translations. Restricting two degrees of freedom in $2 \mathrm{D}$, by either fixing an arbitrary node or the average of some chosen nodes is a trivial step completing the definition of the periodic cell model. 


\subsection{Modeled periodic cell and captured points in Brillouin zone}

A rectangular periodic cell domain $\Omega$ is chosen here for simplicity as it can both represent rectangular and rhombic lattices, however, a generalization to oblique periodic cells should be straightforward by redefining the mappings $R \rightarrow L$ and $T \rightarrow B$ accordingly. As indicated on Figure $3, \Omega$ does not necessarily need to represent an elementary unitcell in the sense of a minimal repeatable unit that defines the microstructure, but it can contain multiple repetitions of the actual unit-cell within the modeled periodic cell. This allows to capture buckling modes with different periodicities in the lattice's Brillouin zone. In the present work, a 2 by 2 repetition of the elementary unit-cell captures in most cases all commonly addressed lattice buckling modes for the studied microstructures. Moreover, the homogenized strain degrees of freedom $\varepsilon_{x y}$ and $\varepsilon_{y x}$ allow for capturing modes of infinite period, corresponding to points at the center of the Brillouin zone.

The periodic homogenization approach adopted here, even if accounting for large deformations, is still limited by the separation of scales requirement. Results obtained by means of a periodic cell model are therefore only relevant for situations where variations of stresses along a unit-cell length are small. It should be noted that even in the case of a unit-cell size small compared to the overall dimensions of a structure, the separation of scales assumption can be violated due to stress concentrations [39]. In such cases, as well as in cases of a large unit-cell compared to the geometry of the structure, one should either use direct simulations of the whole structure as in [40], or higher-order homogenization methods as in [39, 41].

\subsection{Load cases}

The load carrying capacity of isotropic materials under plane strain conditions can easily be represented in the in-plane principal stresses space, based on biaxial loading experiments along the axes of an arbitrary coordinate system. The applicability of this simple description does nevertheless not carry over to materials with a periodic microstructure, which are inherently anisotropic. In this case, the response upon biaxial loading does not only depend on the magnitude of the two imposed normal stress components but also on the relative orientation between principal stress and material symmetry axes. In general, different yield surfaces should be expected for different orientations between loading and material directions.

Available investigations of the load carrying capacity of anisotropic materials often implicitly neglect this orientation dependence. Works accounting for this load orientation dependence typically report yield surfaces in multidimensional stress spaces in terms of both normal and shear stress components, as for instance [19]. However, in order to avoid the often difficult to interpret multidimensional stress spaces, a common simplification is to only consider uniaxial loading spanning the entire space of possible load orientations. In this case, yield limits for uniaxial loading are either reported through yield surfaces in a polar coordinate system, or through inverse pole figures [21]. To ensure a simple and practical presentation of the studied 2D lattice structures, the present work considers two basic load cases.

Biaxial loading. In the first case, biaxial loading is applied in a fixed arbitrarily chosen coordinate system, with different longitudinal to transverse stress ratios. Since all structures investigated in this study have at least a pair of perpendicular symmetry axes $X Y$, an obvious choice is to align the load coordinate system $x y$ with these material principal axes. With stability investigations in focus, compression is imposed along one of the loading axes by prescribing the corresponding longitudinal strain $\varepsilon_{y y}$ in Eq. (2), incrementally. At the same time, a zero longitudinal shear strain condition $\varepsilon_{y x}=0$ is imposed, while the transverse shear strain $\varepsilon_{x y}$ is set as free, i.e. $P_{x y}=0$. Finally, regarding the loading in the transverse direction $x$, Eq. (10) is employed with different positive and negative values for the Cauchy stress ratio $k$, resulting in either compressive or tensile stresses $\sigma_{x x}$, respectively. The resulting loading path corresponds to a straight line in the $\left\{\sigma_{x x}, \sigma_{y y}\right\}$ space for a coordinate system $x y$ at fixed orientation $\alpha=0$.

Rotated uniaxial loading. The load orientation effect neglected in the previous load case is investigated by imposing a uniaxial load along the $x$ direction of the loading coordinate system $x y$ at varying orientation angles $\alpha$ with respect to the material coordinate system $X Y$. In this case, the longitudinal strain $\varepsilon_{x x}$ is prescribed incrementally and the conditions $\varepsilon_{x y}=0$ and $P_{y y}=P_{y x}=0$ are imposed. In general, for an arbitrary angle $\alpha$ the uniaxial loading in $x$ direction leads to normal and shear stress components $\sigma_{X X}, \sigma_{Y Y}$ and $\sigma_{X Y}$ in the material coordinate system $X Y$, that can not easily be illustrated in a 2D diagram as in the previous case. For this load case, yield surfaces are plotted in a polar diagram with the applied stress $\sigma_{x x}$ as the radial coordinate and the orientation angle $\alpha$ as the circumferential coordinate. 


\subsection{Perturbations}

In contrast to eigenvalue buckling analysis which is performed on geometrically perfect structures, direct nonlinear analysis relies on appropriate perturbations or imperfections in order to capture relevant buckling modes. In the present work, three different types of perturbations are applied depending on the microstructure investigated and the type of simulation.

Targeted geometrical perturbations. A standard way of introducing imperfections in a structure is by perturbing node coordinates of the computational mesh, resulting in small geometrical changes. These geometrical modifications are typically chosen according to some buckling mode of interest, known e.g. from a linear eigenvalue analysis. In this manner, it is also possible to trigger one specific buckling mode even if this is not the critical one. For this kind of perturbations, the severity of the imposed imperfection is controlled by the amplitude $a$ of the imperfection. This can be converted to a roughly estimated characteristic misalignment angle $\phi=\arctan a / l_{0}$, where $l_{0}$ is the unit-cell size.

Random geometrical perturbations. Proper random geometrical perturbations are essential for enabling multiple buckling modes, hence also capturing the most critical ones. Especially when dealing with multiscale structures, it is important that imperfections introduced at different length scales are of comparable intensity. Otherwise, the effect of buckling modes in some length scales may either be amplified or suppressed compared to modes in other length scales. Figure 4 illustrates a simple but rather generic approach, followed in the present work, for generating random geometrical perturbations.

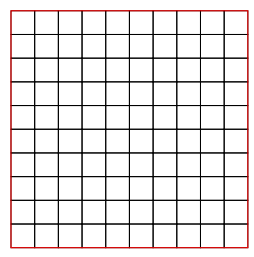

(a) Unperturbed

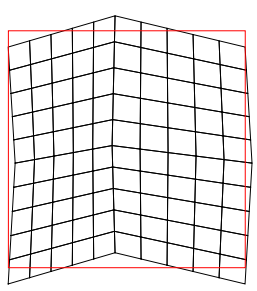

(b) $d=l / 2$

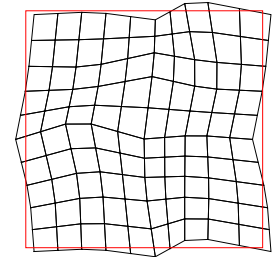

(c) $d=l / 4$

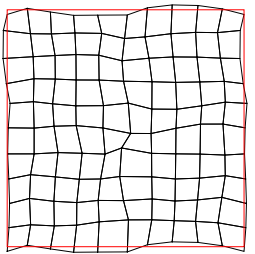

(d) $d=l / 8$

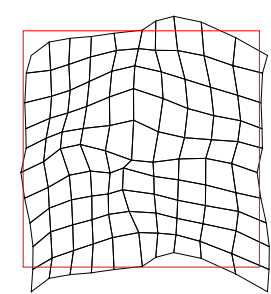

$(\mathrm{e})=(\mathrm{b})+(\mathrm{c})+(\mathrm{d})$

Figure 4: Superposition of random perturbations with different length scales.

In order to introduce imperfections at several wavelengths, different grids of points are defined with spacing $d$ equal to each desired length scale. The points of each grid, which are not necessarily nodes of the computational mesh, are then perturbed in $X$ and $Y$ directions by random amounts with a uniform statistical distribution in the interval $[-d / 2 \tan \phi, d / 2 \tan \phi]$. The nodes of the actual mesh of the microstructure are displaced according to a linear interpolation between the surrounding points on the perturbation grid. By choosing the perturbation amplitude proportional to each wavelength $d$, as defined above, it is ensured that the same characteristic misalignment angle $\phi$ is represented in each perturbation grid, independent of the length scale. Finally the overall perturbation of the microstructure mesh is obtained by superposition of a number of different perturbation grids at different length scales, as conceptually illustrated in Figure 4

Shear load. Since an overall shearing deformation is a common collapse mode for non-isotropic microstructures under compression, it is often convenient to use a small shear load as a perturbation for triggering this kind of collapse. For the magnitude of the imperfection to be comparable to the other two cases, one should ensure that the overall shear angle of the microstructure under the perturbation load is of the same order as $\tan \phi$.

\section{Interpretation and presentation of results}

In a direct nonlinear analysis of a periodic microstructure, quantities of interest such as applied homogenized stress and resulting average strain components will often vary in a complex and non-proportional manner. Moreover, the distinction between a sharp buckling initiation and a progressively softening response can often be rather diffuse when kinematic and material nonlinearities are inherently taken into account. These aspects underline the importance of a systematic analysis and presentation of the obtained results, addressed in detail in the present section. 


\subsection{Stress normalization}

In order to obtain comparability between calculated stress limits for different microstructures and volume fractions, it is necessary to employ a normalization that is independent of the type of the microstructure and compensates for any known bounds at the low volume fraction limit. In [28], the characteristic stress $E /(t / L)^{3}$ was used as a reference value for the normalization of stresses, where $E$ is the material Young's modulus, $t$ is the thickness and $L$ is the length of the lattice struts. This characteristic stress definition is based on the Euler buckling limit of double pinned bars of the given slenderness.

Although this is a valid approach when looking at one specific lattice type, different types of lattices made of struts of the same slenderness will in general occupy different volume fractions. This means that $t / L$ is not an appropriate parameter for benchmarking different lattices in terms of material utilization. In that sense, instead of the strut slenderness, the material volume fraction $V^{*}$ should actually be used as the basis for defining a characteristic stress. In this fashion, comparability is achieved for generic microstructures, even including a multiscale topology, because the volume fraction $V^{*}$ is always unambiguously defined in contrast to the parameter $t / L$ that assumes a truss-like structure.

Noticing the approximate relation $V^{*} \approx 2 t / L l$, valid for the low volume fraction regular square lattice studied in [28], we suggest a reference stress for normalization purposes, defined as

$$
\sigma^{*}=E /\left(V^{*} / 2\right)^{3} \text {. }
$$

A high level of universality and comparability of the presented results can then be achieved by reporting dimensionless stress quantities

$$
\bar{\sigma}=\sigma / \sigma^{*}
$$

instead of the Cauchy stress components from Eq. (4). This choice allows for a direct comparison of microstructures of all shapes and volume fractions, even though the reference stress $\sigma^{*}$ has a physical interpretation for the regular square lattice only. The adopted normalization compensates for the known volume fraction dependence of buckling limits for stretch dominated slender microstructures, which means that any additional dependence of the normalized stress $\bar{\sigma}$ on $V^{*}$ is an additional finite volume fraction effect.

\subsection{Definition and use of a collapse criterion}

Central to the present study is the determination of loss of stability of a microstructure under compressive load. In contrast to linear buckling analysis, where such a stability limit is mathematically defined in all cases, the actual nonlinear response of a microstructure can transition towards a complete loss of stiffness without going through a bifurcation point. Even in cases where a real bifurcation exists, this will typically still be smoothened by the adopted geometrical perturbations. With practical requirements regarding the load carrying capacity of a microstructure in mind, it is useful to provide a universal and practical definition of a point of bucking or failure. Here, the choice is made to define failure by considering a threshold for the tangential stiffness measure defined as

$$
\mathcal{K}=\frac{\|\dot{\boldsymbol{\sigma}}\|}{\|\dot{\varepsilon}\|} \approx \frac{\|\Delta \sigma\|}{\|\Delta \varepsilon\|}
$$

where $\Delta \sigma$ and $\Delta \varepsilon$ are respectively increments of the stress and strain tensor within a static load step. The use of the Frobenius norm for the definition of this effective tangent stiffness is a natural choice as it is invariant with respect to fixed rotations of the chosen frame of reference.

Based on the effective tangent stiffness defined above, a practical and generic failure criterion can easily be defined as

$$
\mathcal{K}<\eta E V^{*}
$$

where $E V^{*}$ corresponds to the upper Hashin-Shtrikman bound with regard to Young's modulus, and $\eta$ is a threshold parameter that specifies the minimum acceptable stiffness as a fraction of the theoretical upper bound. By defining failure at a small but non-zero level of stiffness, the adopted criterion encompasses both buckling and softening in a unified manner, rather than capturing only classical bifurcation buckling. The exact value of the threshold $\eta$ is not crucial in case of bifurcational buckling in which the stiffness of the structure drops drastically from its maximum 
towards a small residual stiffness. Only in cases of progressive softening, there is a significant dependence of the failure line on the chosen threshold value. Throughout this work a value of $\eta=0.1$ is adopted.

To illustrate the use of the failure criterion proposed in Eq. (14), Figures 5 and 6 show typical simulation results for a square lattice and the two load cases described in section 2.3. During the simulations, the compressive longitudinal strain was incremented until a specified final strain using variable step sizes to ensure a bounded norm of displacement field increments. Biaxial loading results at different fixed stress ratios $k$ are shown in Figure 5 . Using the failure criterion of Eq. (14), the stress state at which failure occurs is found individually for every ratio $k$ and finally a polynomial fit is used to obtain the resulting failure line, shown in the figure as a gray solid line. It becomes evident in the plot including the strain axis $\varepsilon_{Y Y}$, how the adopted criterion captures the observed kink point along the plotted curves.

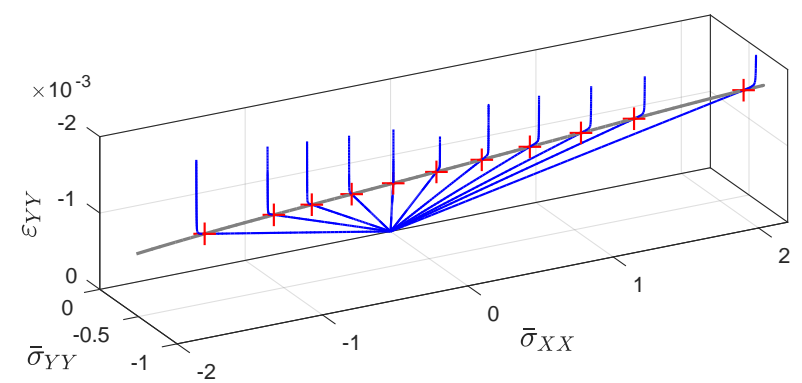

(a) Angled view including the strain axis.

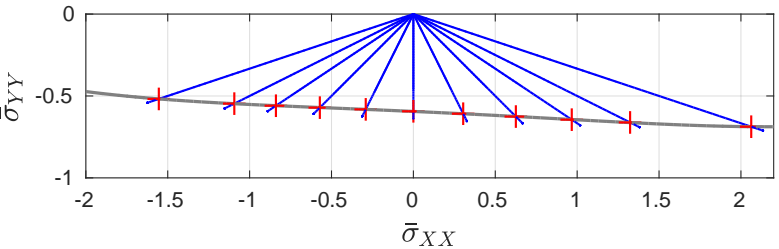

(b) Top view showing only the stress space.

Figure 5: Stress-strain curves in two-dimensional normalized stress space illustrating the biaxial loading procedure on the example of the square lattice. The loading paths are straight lines in Cauchy stress space, the red markers indicate the detected buckling points and the gray line is the resulting fitted failure line.

Figure 6 shows corresponding results for uniaxial loading with the load direction $x$ oriented at different angles $\alpha$. The plotted failure points define a failure line in the polar diagram of uniaxial stresses that describes the anisotropic buckling behaviour of the considered square lattice. Exploitation of symmetries in the periodic cell reduces the range of required angles significantly.

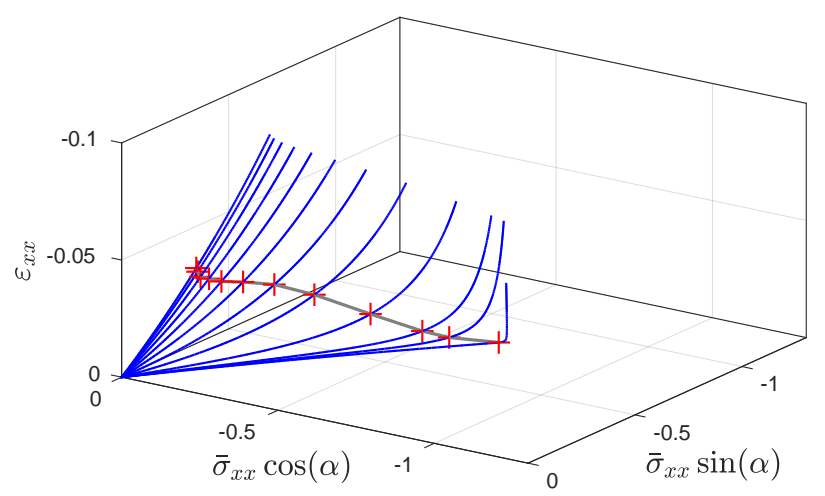

(a) Angled view including the strain axis.

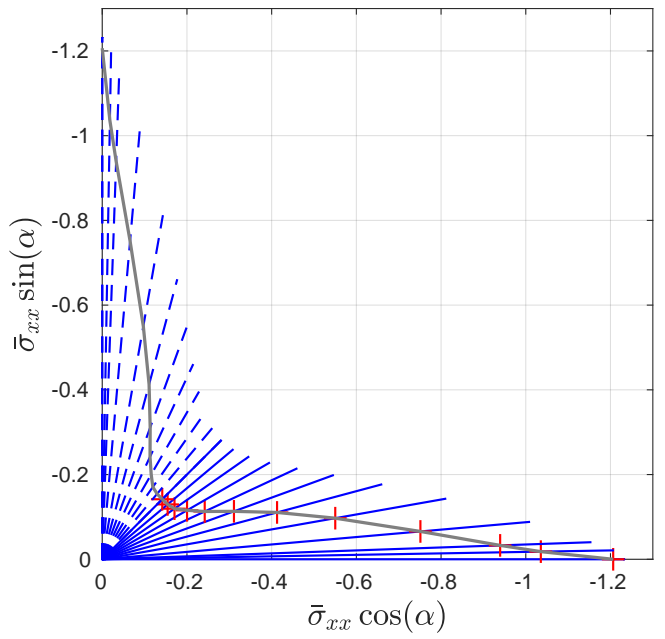

(b) Top view showing only the stress space.

Figure 6: Stress-strain curves for uniaxial loading at different angles to illustrate the uniaxial loading procedure on the example of the square lattice. The red markers indicate the detected failure points. The dashed lines are reconstructed by exploiting the symmetry of the structure. 


\section{Results and Discussion}

This section presents results from numerical studies performed according to the methodology described above, applied on three very common lattice structures at different volume fractions and one optimized hierarchical structure from the literature, all summarized in Table 1. The table also shows the orientation of each microstructure within the $X Y$ frame with $X$ corresponding to the horizontal and $Y$ to the vertical direction. For biaxial loading, results are presented in terms of failure lines in the two-dimensional principal stress space, obtained according to the failure criterion from Eq. (14). Results for the case of rotated uniaxial loading are plotted in polar coordinates instead, with the radius corresponding to the applied stress $\sigma_{x x}$ and the angle coordinate corresponding to the orientation angle $\alpha$ between the loading direction axis $x$ and the microstructure axis $X$. The normalized tangent stiffness $\mathcal{K}$ is shown in this kind of polar diagrams as a colormap plot, combined with the failure line obtained according to Eq. (14) for $\eta=0.1$. The outer contour of the colormap plots corresponds to an arbitrary chosen maximum strain applied during the simulations, and accepts therefore no specific interpretation as the failure line does.

Table 1: Investigated microstructures with different volume fractions. The slenderness ratio $L / t$ of constituent bars is based on center to center distances between bar intersections. Unit-cells are defined through the black frames in the row for $V^{*}=0.3$ and the number of unit-cells inside the modeled periodic cell are given in parenthesis in the first row.

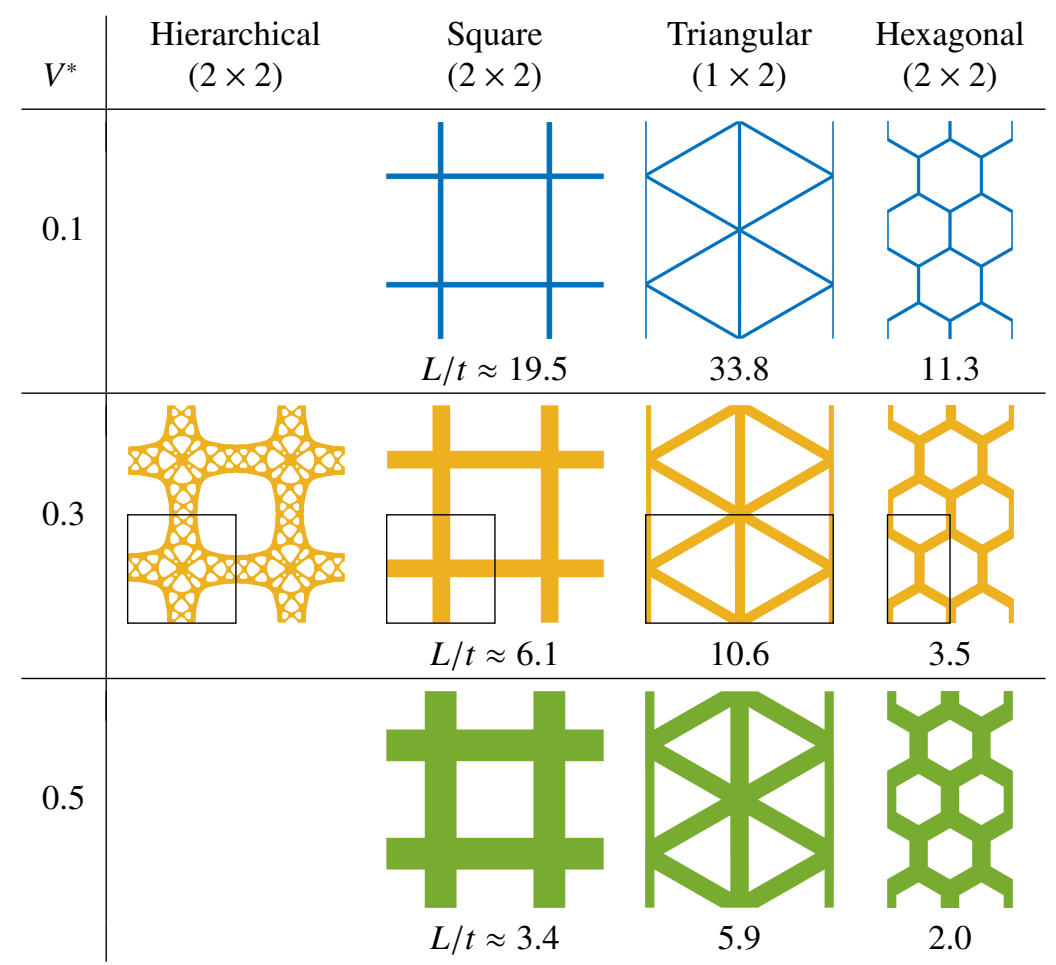

\subsection{Square Lattice}

The square lattice owes its popularity mainly to its simplicity and has been subject of a large number of investigations, with compressive stability limit lines at the limit of small volume fractions, reported in [28]. The biaxial loading results shown in Figure 7 extend this previous result with failure lines for finite volume fractions for the two most critical buckling modes from the aforementioned study. The post-buckling deformation patterns corresponding to the two modes are also included in the figure. All failure lines are based on a polynomial fit to the actual data points, which are marked on the figure. 


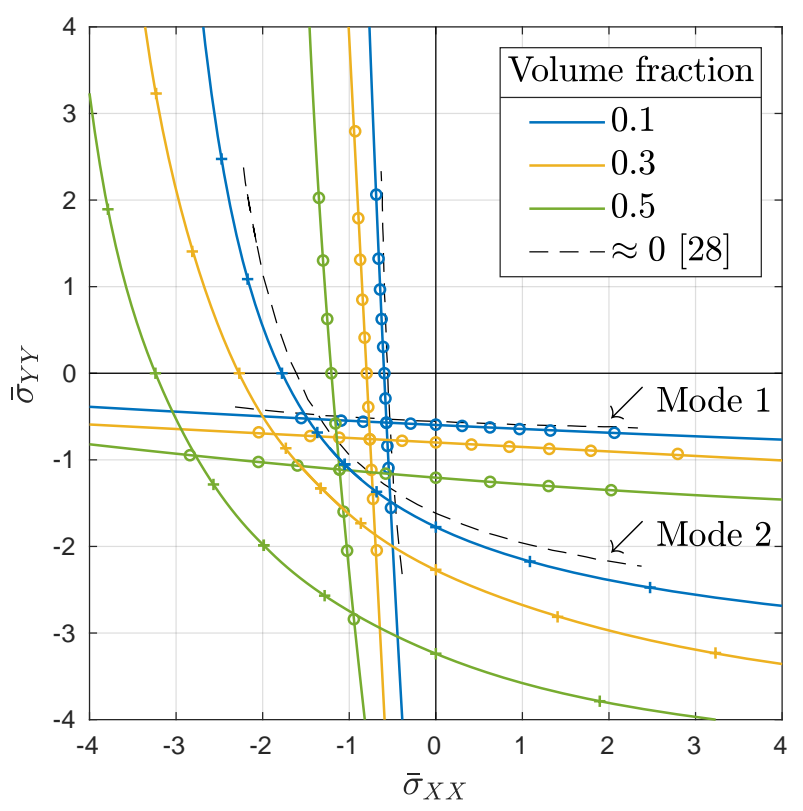

(a) Failure lines

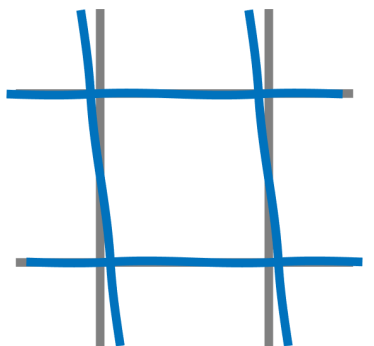

(b) Mode 1

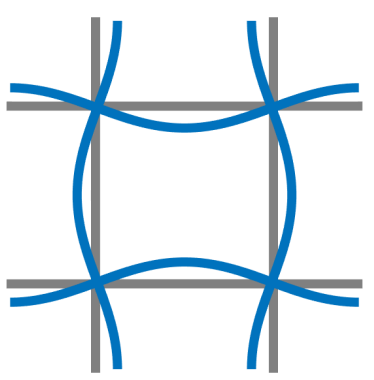

(c) Mode 2

Figure 7: Regular square lattice failure lines in two-dimensional stress space (a) for the two illustrated deformation modes (b,c), shown for $V^{*}=0.1$. Markers represent simulation results, while lines correspond to a polynomial fit. Circle and plus sign markers respectively represent failure of mode 1 and 2. The black dashed failure lines are the reference solution from [28] for the low volume fraction limit.

Buckling mode 1. This mode, illustrated in Figure 7p, corresponds to shear deformation of the entire periodic cell in the sense of either $\varepsilon_{y x}$ or $\varepsilon_{x y}$ strains, depending on whether $x$ or $y$ is considered as the main compressive loading direction. The corresponding two failure lines which intersect on the equibiaxial load path $\sigma_{x x}=\sigma_{y y}$ due to symmetry, define the critical inner failure envelope for the structure, with the shear deformation perpendicular to the major compression direction being always critical. The fitted failure lines are almost straight with a slight slope, which increases with volume fraction, indicating that tensile transverse stresses delay buckling initiation while compressive transverse stresses promote buckling. However, especially for slender structures, the effect is not very pronounced and the buckling initiation for this mode mainly depends on the longitudinal compressive stress. The failure lines plotted for different volume fractions demonstrate the strong additional effect of increased volume fractions compared to the theoretical dependence through Eq. (11), valid only for very low volume fractions. For comparison, corresponding results for $V^{*} \rightarrow 0$ from [28] are included in the figure, adapted though to the material properties and the plane strain condition assumed here. The obtained results for the lowest volume fraction of $10 \%$ are rather close to the included reference results for very slender structures. There are however visible differences already at this low volume fraction, especially for the second buckling mode. Deviations are partly due to a non-vanishing effect of the strut intersection regions, but also due to geometrical and material nonlinearities, accounted for in the nonlinear continuum mechanical model. For the remaining two higher volume fractions, the deviations from [28] are large.

Buckling mode 2. Although the buckling mode presented above is in general expected to be the most critical one, [28], boundary conditions may prevent its occurrence so that the second most critical mode shown in Figure 7k becomes important. For provoking this mode within the nonlinear analysis employed, a targeted geometrical perturbation of corresponding shape was used with an amplitude of $10^{-4} l_{0}$ for $V^{*}=0.1$ and $5 \cdot 10^{-4} l_{0}$ for $V^{*}=0.3$ and 0.5 . This second buckling mode can occur independent of whether the main compressive load is in $X$ or $Y$ direction and there is therefore only one continuous failure line in the whole stress space. As expected, the buckling resistance for this mode is always larger compared to the shearing mode described above. A strong presence of finite volume effects is also observed in this case with the most dense structure for $V^{*}=0.5$ being approximately twice as strong as predicted by the low volume fraction approximation. The buckling limits of the two modes are closest to each other along the equibiaxial load line but still, the limit of mode 2 is 1.5 to 2 times higher than for mode 1 . 
Buckling performance for rotated uniaxial loading. Figure 8 shows results for uniaxial loading of varying orientation according to section 2.3, demonstrating a strongly anisotropic load carrying capacity. At the left of the figure, the normalized tangent stiffness $\mathcal{K} /\left(E V^{*}\right)$ is shown for $V^{*}=0.5$, with the plotted solid contour line corresponding to the failure line defined by the adopted threshold value of 0.1 . At small loads in direction parallel to the struts, the normalized initial tangent stiffness is in the range of 0.6 to 0.7 for all volume fractions.

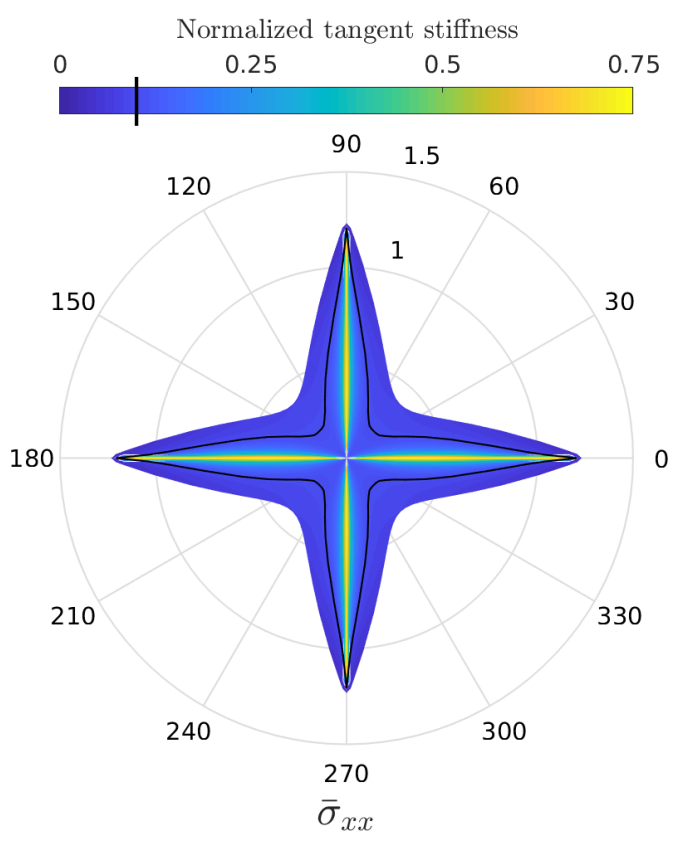

(a) $V^{*}=0.5$

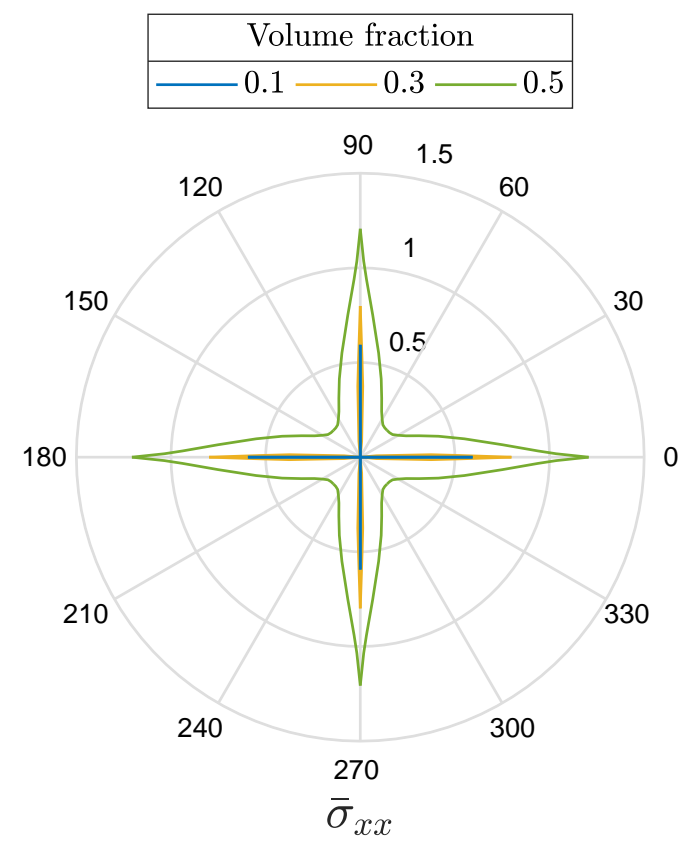

(b) Failure lines

Figure 8: Normalized tangent stiffness under uniaxial load, with 0.1 threshold line for the regular square lattice with $V^{*}=0.5$ (a) and failure lines for different volume fractions (b).

The failure lines plotted in Figure 8 reveal the poor performance of the regular square lattice microstructure in any direction other than parallel to the lattice bars. Even for the highest volume fraction of $V^{*}=0.5$, it exhibits for load orientation angles $\alpha$ between $30^{\circ}$ and $60^{\circ}$ less than $10 \%$ of its maximum load carrying capacity which occurs along the lattice bars, i.e. at $\alpha=0^{\circ}$ or $90^{\circ}$. At most intermediate load orientations, the structure does not show buckling at all, but instead undergoes a shear deformation with continuously decreasing stiffness, eventually hitting the set threshold value. This effect is more pronounced for low density structures due to the diminishing bending stiffness.

The discrete load orientations used for generating these results correspond to intervals of $5^{\circ}$ for angle $\alpha$ from $0^{\circ}$ to $45^{\circ}$ and two additional cases with $\alpha=1^{\circ}$ and $2^{\circ}$. Perturbations were only necessary for $\alpha=0^{\circ}$, when the load is applied in the direction of the lattice bars. The latter is a case already contained in the aforementioned biaxial loading case, where the global shear mode is critical, hence shear load perturbations are applied here, as well.

\subsection{Triangular lattice}

Another example of a widely used and extensively studied microstructure is the triangular lattice which exhibits an isotropic initial stiffness. The nonlinear analysis results shown in Figure 9, demonstrate however, how this isotropy is lost due to geometrical nonlinearities, with the failure lines illustrating how the buckling performance under uniaxial load varies as a function of the load angle. Moreover, a strong finite volume fraction effect on the expected buckling limit is also observed here. While all triangular lattice structures with volume fractions below 0.1 are expected to have failure lines very close to the one plotted for the 0.1 case, for larger volume fractions the deviations are large.

Interestingly, the microstructure volume fraction does not only expand the plotted failure lines radially, but it also affects their shape. Triangular lattice structures with volume fractions 0.1 and 0.3 exhibit their highest load bearing 
capacity at angles of $0^{\circ}$ and $\pm 60^{\circ}$, i.e. along directions perpendicular to the lattice bars, while their lowest buckling resistance is observed in directions parallel to the lattice bars. However, at $V^{*}=0.5$, the lattice has almost isotropic buckling resistance, while at even higher volume fractions, the anisotropy pattern changes further, with the direction parallel to lattice bars becoming the strongest, which can be seen from the additional plotted failure line for $V^{*}=0.7$.

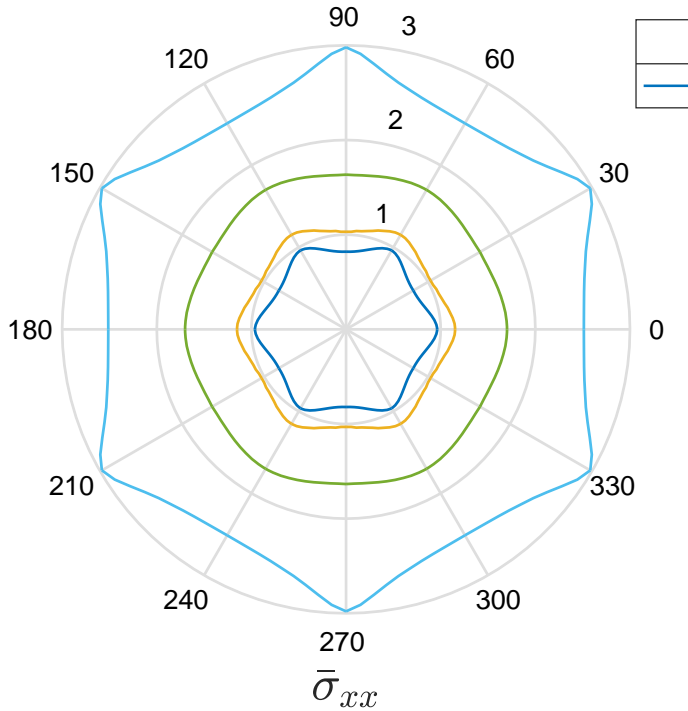

(a) Failure lines

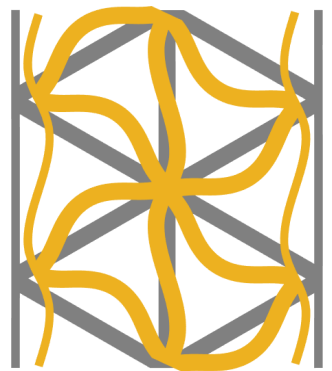

(b) $\alpha=0^{\circ}$

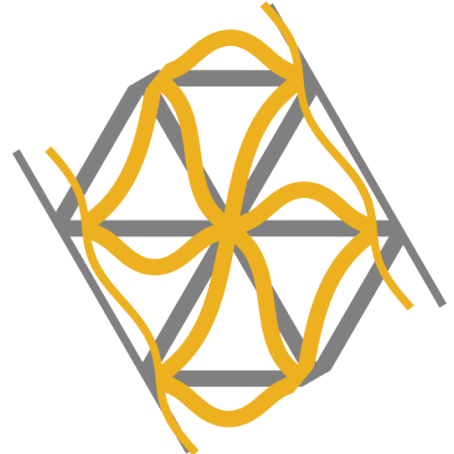

(c) $\alpha=30^{\circ}$

Figure 9: Failure lines for the triangular structure at different volume fractions under uniaxial load (a), and deformed structures for different loading angles $\alpha$ at $V^{*}=0.3(\mathrm{~b}, \mathrm{c})$.

Figures $9 \mathrm{~b}$ and $9 \mathrm{c}$ show the post-buckling deformed microstructures for $V^{*}=0.3$ with $0^{\circ}$ and $30^{\circ}$ rotations, respectively, under uniaxial loading in the $x$-direction. Plotting the normalized tangent stiffness is omitted, as for this structure it exhibits rather high values in the range of $\mathcal{K} \approx 0.4$ to 0.8 in almost the entire region inside the plotted failure lines, with a sharp collapse on the failure lines. The presented results were obtained by loading at discrete orientation angles $\alpha$ from $0^{\circ}$ to $30^{\circ}$ at intervals of $3^{\circ}$ and two additional cases with $\alpha=1^{\circ}$ and $29^{\circ}$. In theory, perturbations are only required for loading directions of $0^{\circ}$ and $30^{\circ}$, at which the symmetry of the structure would prohibit the occurrence of the otherwise observed non-symmetric buckling modes. Nevertheless, the shown results are based on consistently applied random geometrical perturbations including four wave lengths ranging from $0.2 l_{0}$ to $l_{0}$ and a characteristic misalignment angle $\phi \approx 0.06^{\circ}$ for $V^{*}=0.7$ and $\phi \approx 0.02^{\circ}$ for all other cases. Since the critical mode for this structure is known, targeted perturbations could have been applied as well.

\subsection{Hexagonal lattice}

The last classical periodic microstructure considered is the hexagonal lattice, which seems to be one of the most studied microstructures in the available literature. It is also very widespread in real applications, despite of its wellknown low shear modulus. Figure 10 shows the normalized tangent stiffness under uniaxial load in varying orientations for $V^{*}=0.3$ and $V^{*}=0.5$, including the corresponding failure lines for the same stiffness threshold as in all previous cases. These lines are compared directly to each other in Figure 11 which also shows post-buckling deformation plots for uniaxial compression at $0^{\circ}$ and $30^{\circ}$. The results shown for this structure were obtained at the same loading angles as for the triangular lattice but without any perturbations. For the uniaxial loading considered here, no crossing of a bifurcation point is expected during the loading procedure and therefore both perturbed and unperturbed structures appear to behave in the same manner.

The plots in Figures 10 and 11 show the anisotropic failure behavior of the hexagonal structure, with the highest stiffness and load carrying capacity observed at $\alpha= \pm 30^{\circ}$ and $90^{\circ}$, i.e. in directions parallel to the lattice bars, while stiffness and load capacity attain their minimum for uniaxial compression perpendicular to the lattice bars. For increasing volume fraction, the failure behavior becomes more isotropic. For $V^{*}=0.5$ for example, the variation 


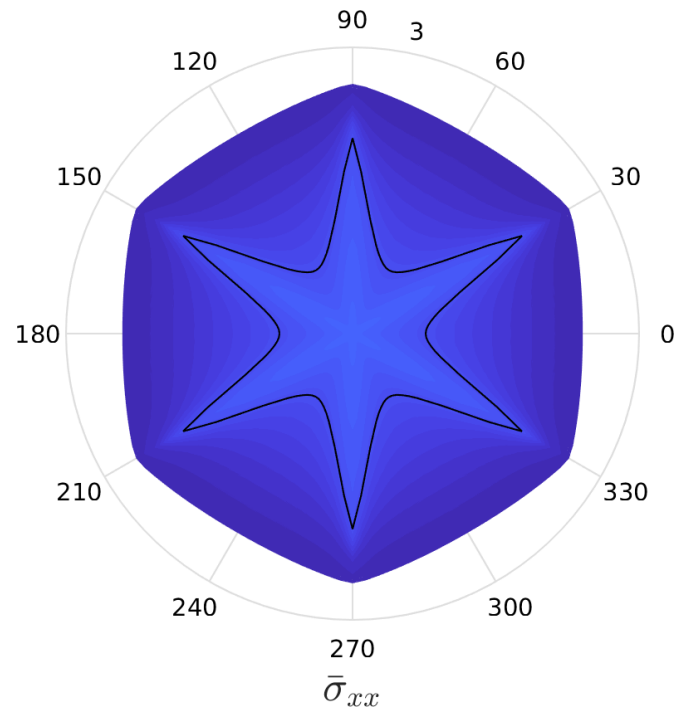

(a) $V^{*}=0.3$

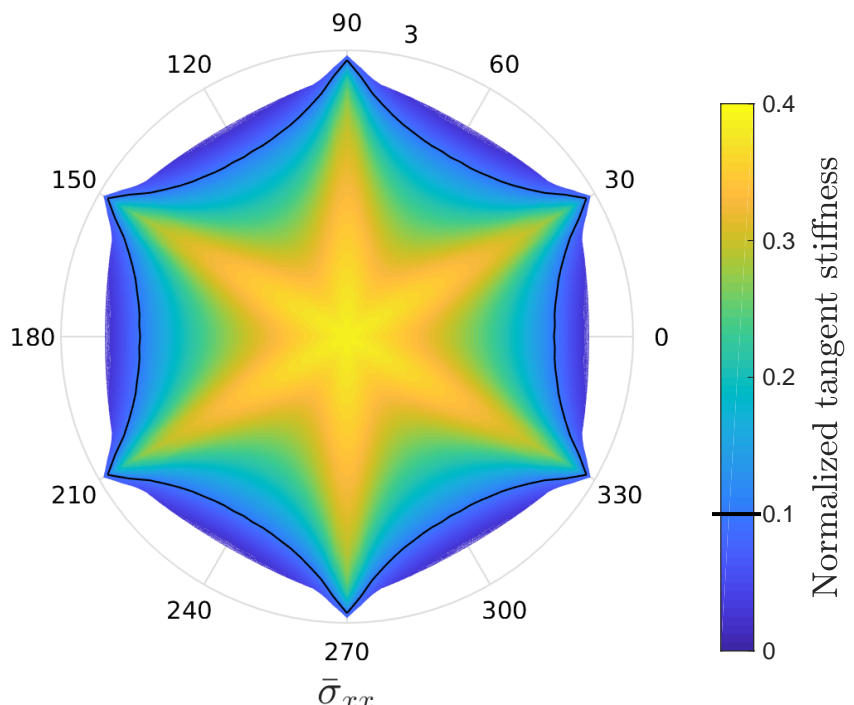

(b) $V^{*}=0.5$

Figure 10: Normalized tangent stiffness with 0.1 threshold line for the hexagonal lattice structure at different volume fractions under uniaxial load.

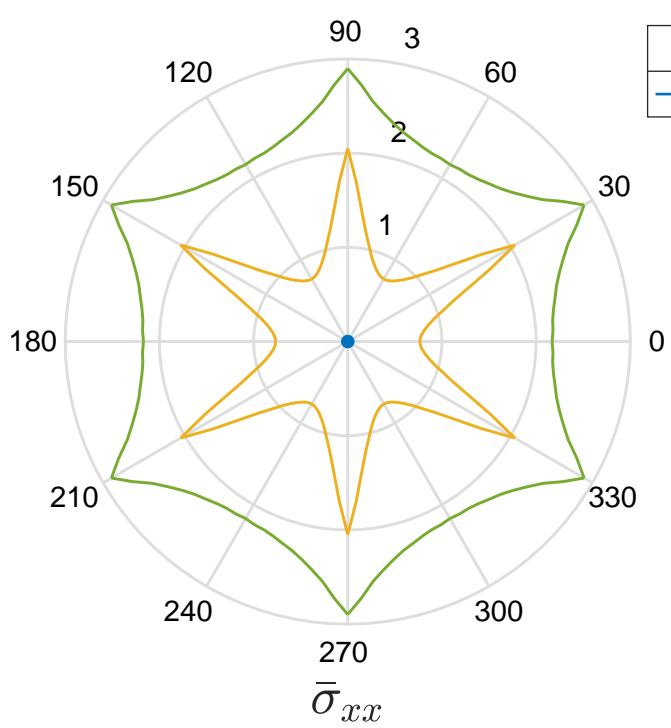

(a) Failure lines

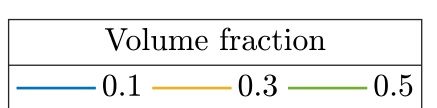

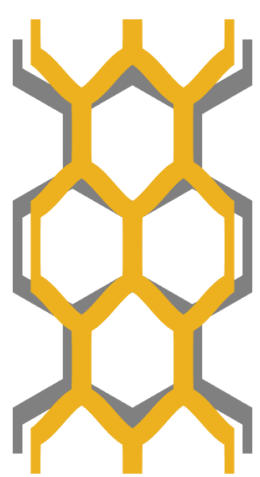

(b) $\alpha=0^{\circ}$

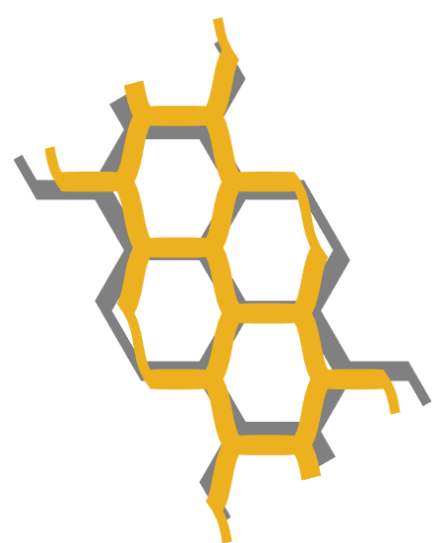

(c) $\alpha=30^{\circ}$

Figure 11: Failure lines for the hexagonal structure at different volume fractions under uniaxial load (a), and deformed structures for different loading angles $\alpha$ at $V^{*}=0.3(\mathrm{~b}, \mathrm{c})$.

between the maximum and minimum failure limit in any direction is just around $25 \%$, while this variation is around $60 \%$ for $V^{*}=0.3$. However, unlike the triangular structure, no radical change in the anisotropy pattern is observed.

At the volume fraction of 0.1 , the hexagonal structure has an initial tangent stiffness below the adopted stiffness threshold $\eta=0.1$ and thus its failure line collapses to a single point, expressing the fact that at the lower volume fraction limit the stiffness of this lattice structure vanishes. Independent of the volume fraction, the tangent stiffness decreases gradually with increasing load, indicating that the structure in most cases looses its stiffness through a progressive softening rather than a sharp buckling collapse. As a consequence, the obtained failure lines for this case depend significantly on the threshold value $\eta$. 


\subsection{Hierarchical Microstructure}

In a recent work, periodic microstructures were designed using topology optimization for maximizing the lowest buckling limit under a single given stress state with principal stresses of fixed ratio and fixed orientation [32]. As demonstrated by the band-diagrams presented in [32], the optimization procedure leads to multiple buckling modes clustered at the lowest buckling load. This rather uncommon and unexplored situation, naturally raises some uncertainty on whether the presence of several simultaneous buckling modes could lead to a more extreme and unusual nonlinear buckling and post-buckling behavior. To address this question, one of the designs from the aforementioned work is evaluated in this subsection in the same nonlinear analysis framework used for the three hitherto discussed lattice structures.

The specific design considered, is the one obtained for maximizing buckling resistance of a square lattice under compressive equibiaxial loading. Figure 12 shows the geometry and the discretization of the considered microstructure with a volume fraction of approximately $V^{*}=0.3$. The large number of potential buckling modes due to the hierarchical nature of the structure motivates the choice of a random geometrical perturbation that will excite a wide range of buckling modes at different wavelengths. The results presented below cover both the unperturbed geometry and the case of a random geometrical perturbation according to section 2.4 with a characteristic misalignment angle $\phi \approx 0.06^{\circ}$ including four wave lengths from $0.2 l_{0}$ to $l_{0}$.
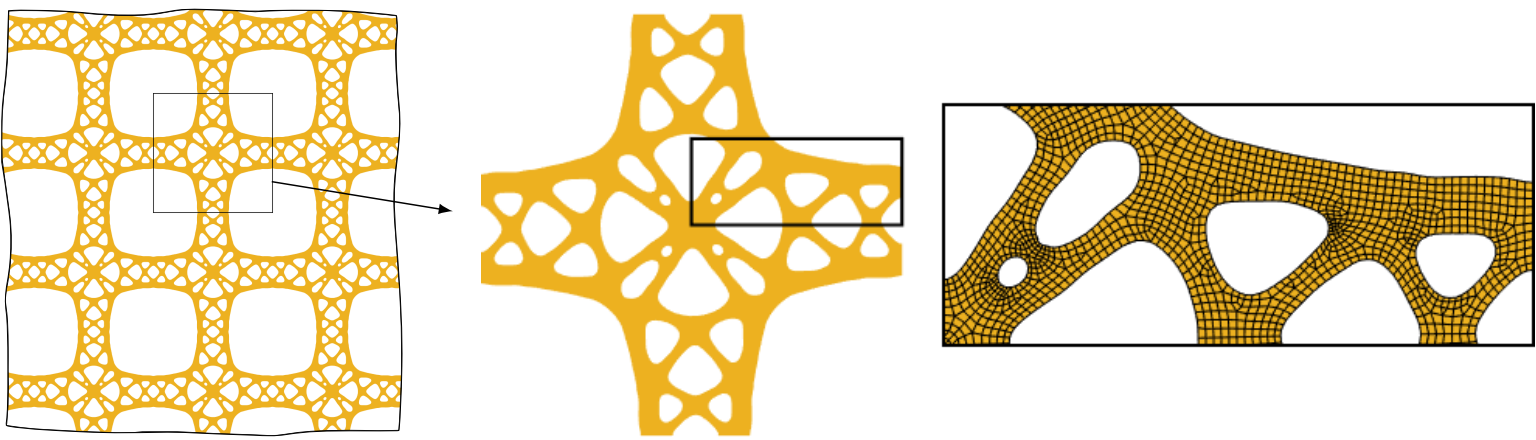

Figure 12: Hierarchical square unit-cell and closeup view of the discretization.

Buckling resistance for biaxial loading. The hierarchical structure is initially analyzed for its buckling performance under biaxial loading along the lattice symmetry axes. Failure lines are obtained both for the original Poisson ratio of 0.4 as well as for the case of a Poisson ratio equal to zero and presented in Figure 13. Corresponding results from a linear buckling analysis according to [32], but for the material parameters used here, are also included for comparison. Moreover, the failure line for the regular square lattice under biaxial loading from Figure 7 is repeated in Figure 13 to put the performance of the optimized hierarchical structure into perspective.

A first observation is that the impact of random geometrical perturbations, investigated in the case for $v=0.4$, is only visible in the region close to equibiaxial compressive loading, cf. points A. In other regions, the failure lines of the unperturbed and the perturbed geometries coincide. In general, due to the clustering of multiple buckling modes at the critical load and the randomness of the perturbations, the shown failure lines do not necessarily correspond to one specific mode, but they rather represent the critical inner envelope of all possible modes satisfying the periodic boundary conditions of the adopted $2 \times 2$ periodic cell.

A second important observation concerns the actual performance of this microstructure compared to the predictions of a linear analysis. To demonstrate the nonlinear effects, the linear analysis from [32] was redone for the plane strain condition, adopted here, and for Poisson ratios of $v=0.0$ and 0.4 , and expressed in terms of true stresses in order to obtain comparability with the nonlinear analysis in Figure 13 For both investigated Poisson ratios, the linear analysis underestimates the buckling strength by approximately $22 \%$ for $v=0.4$ and $16 \%$ for $v=0$ in case of the loading state B. Noting that nonlinear effects are much less pronounced for the regular square lattice structure that fails at much lower strains, the nonlinear analysis demonstrates an even larger gain in buckling resistance through the optimized hierarchical structure, than based on the linear analysis carried out in [32]. Regarding the effect of the material Poisson ratio it should also be noted that the additional strengthening predicted by the nonlinear analysis 


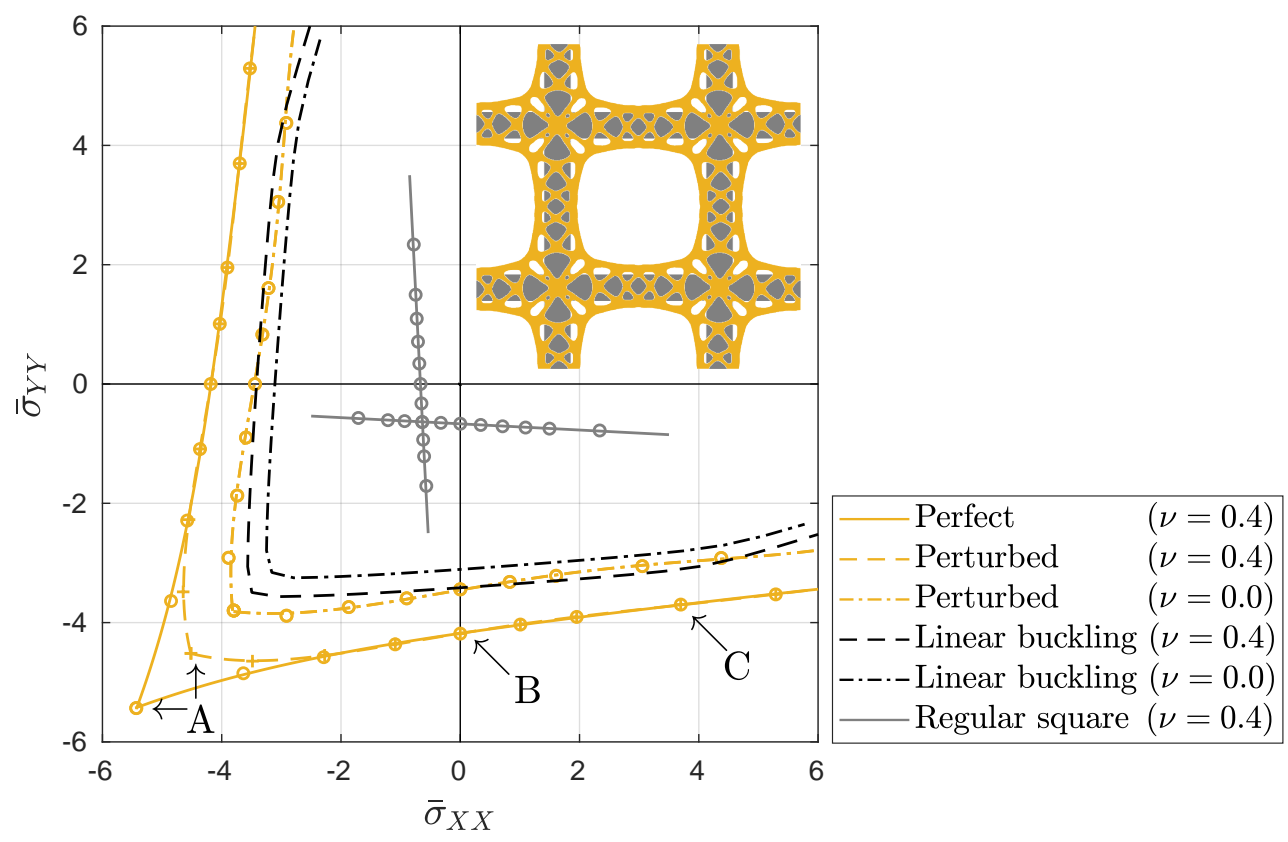

Figure 13: Failure lines for the hierarchical structure and the regular square lattice under biaxial load. The solid yellow line refers to a perfectly symmetric structure without perturbation, while the dashed line is obtained by random perturbations.

for $v=0.4$ compared to $v=0$, is due to thickening of compressed struts members that can only be captured by a nonlinear analysis.

Figures 14 and 15 show stress-strain curves for the two load cases marked in Figure 13 with A and B, respectively. For the equibiaxial load case A, shown in Figure 14, a comparison between the results for the perfect and perturbed structures demonstrates the presence of a distinct instability limit which is not captured with the unperturbed structure. However, for the uniaxial load case B, shown in Figure 15. no distinct failure limit can be observed. Both the perfect and the perturbed geometries approach a vanishing stiffness in the same manner. Nevertheless, two distinct postbuckling behaviors can be observed in Figure 15 , with the perturbed structure exhibiting a collapse-like response not captured with the perfect structure.

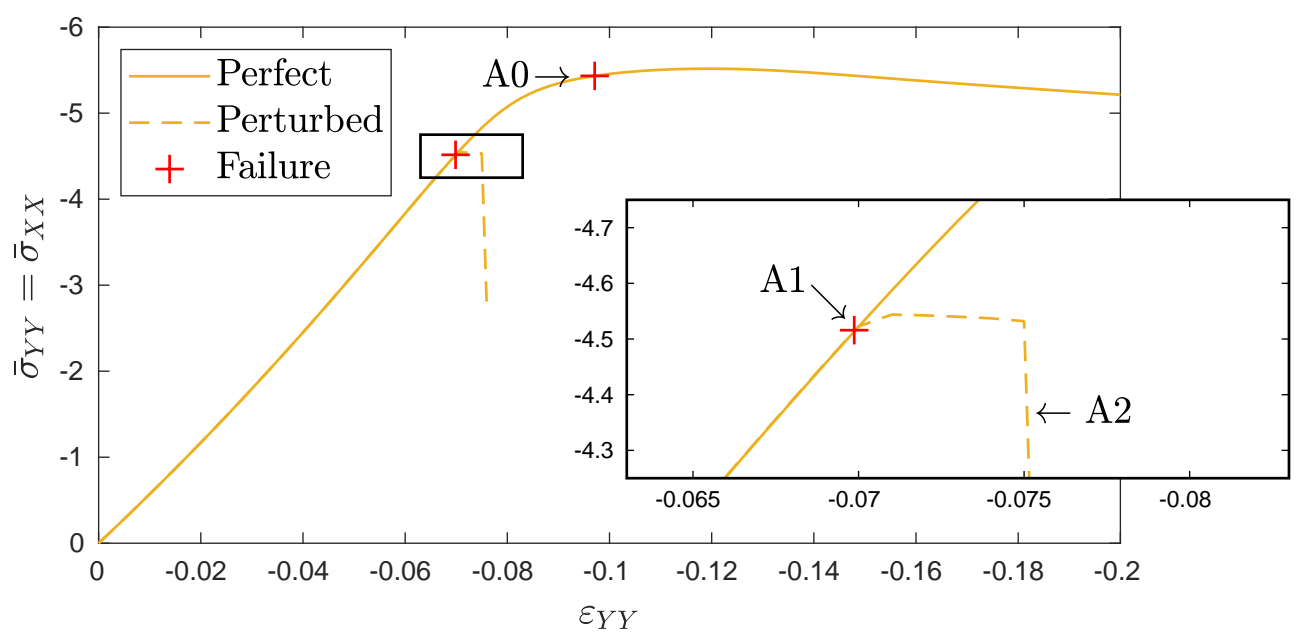

Figure 14: Stress-strain curves for the equibiaxial stress state, marked with A on Figure 13 for both the perfectly symmetric and randomly perturbed hierarchical structure. The red plus sign denotes the point of failure corresponding to the adopted tangent stiffness criterion. The response curve for the perturbed structure has been truncated due to the onset of self-contact not captured by the model. 


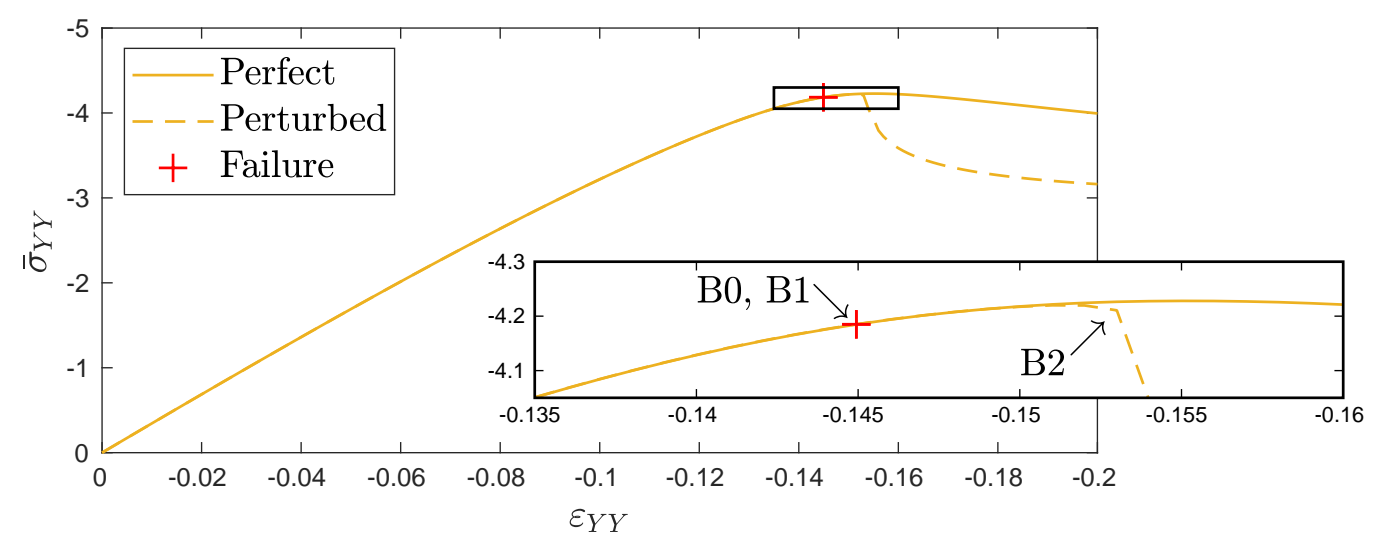

Figure 15: Stress-strain curves for the uniaxial stress state, marked with B on Figure 13 for both the perfectly symmetric and randomly perturbed hierarchical structure. The red plus sign denotes the point of failure corresponding to the adopted tangent stiffness criterion.

Table 2 provides plots of the deformed hierarchical structure at the detected failure limit, for the three load cases A, B and C, labeled in Figure 13, as well as post-buckled structures for cases A and B. For the equibiaxial load case $\mathrm{A}$, the distinct buckling limits between the perfect structure at A0 and the perturbed structure at a lower level at A1, observed in Figure 14, are also represented in the distinct buckling modes shown in Table 2. While a local and symmetric buckling is observed for the perfect structure, the perturbed one buckles in a global shear mode that could not be otherwise captured for the perfectly symmetric geometry. The shear buckling mode captured by the perturbed structure transitions to a localized buckling mode A2 in the post-buckling region, corresponding to the abrupt drop in stress observed at the corresponding point in Figure 14. For load cases B and C, the observed deformation modes leading to a vanishing stiffness are independent of whether perturbations are present or not. As shown in Figure 15 for load case B though, a perturbed geometry allows to capture a subsequent rather abrupt drop in stress at point B2, which corresponds to a switch to a different buckling mode, shown in Table 2 Unlike case B, the initial local buckling that causes the structure to loose its stiffness in load case $\mathrm{C}$, does not transition into any other buckling modes for further increased compression. The two examples, shown for cases A and B with one buckling mode transitioning into another one, causing more or less abrupt stress drops, is a characteristic of hierarchical structures, where buckling can occur at different scales. 
Table 2: Buckling modes for the perfect and perturbed hierarchical structure at different normal stress ratios at the stage of pre- and post-buckling. The labels refer to the ones on Figures 13,14 and 15

Perfect

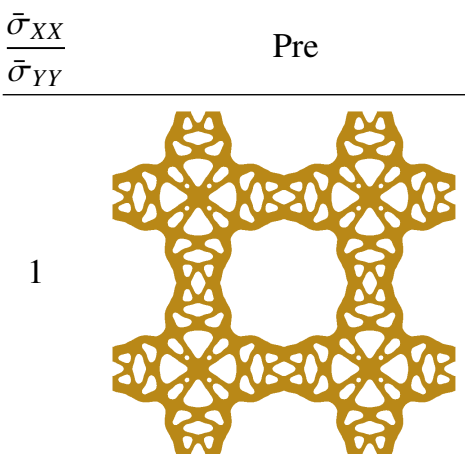

A0

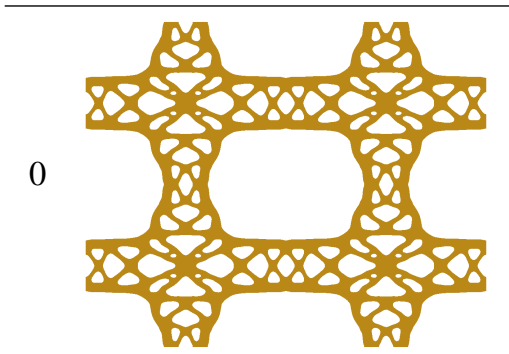

B0
Perturbed

Pre

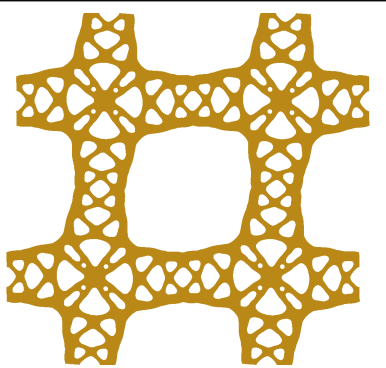

A1

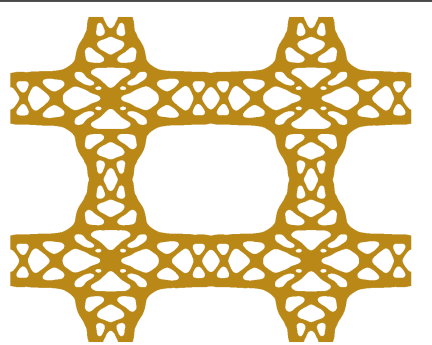

B1

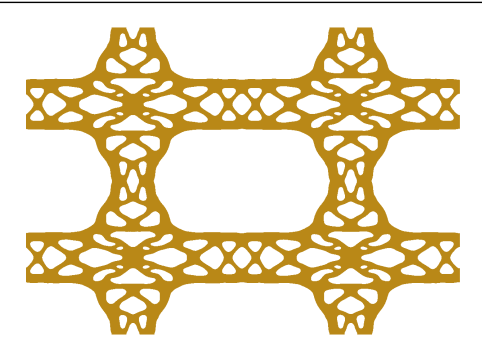

$\mathrm{C}$
Post

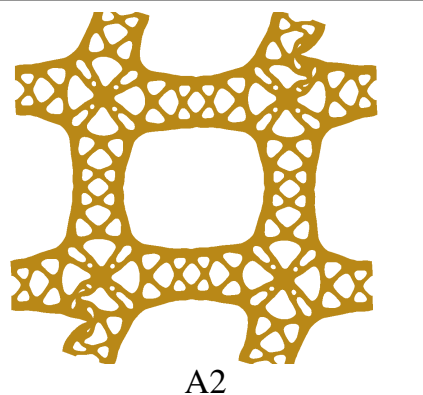

A2

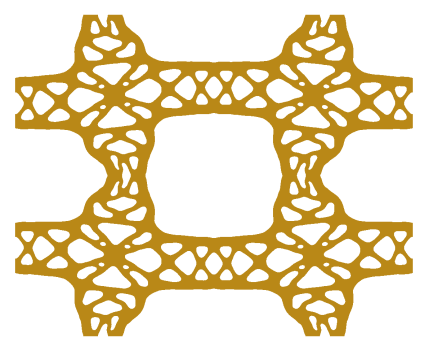

B2

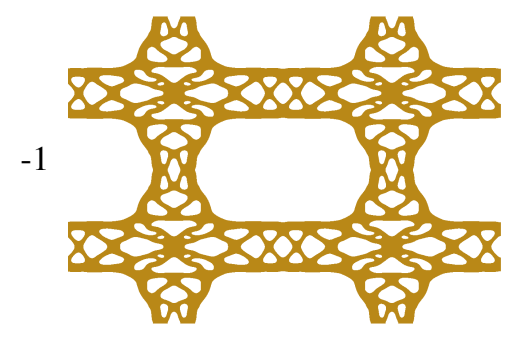

C

Buckling performance for rotated uniaxial loading. Even though the studied hierarchical structure design is optimized for pure equibiaxial load, the results from Figure 15 show that it also performs well under uniaxial load, at least for loading parallel to the unit-cell symmetry axes. The performance of the hierarchical structure under uniaxial loading in different orientations is further investigated in Figure 16, based on simulations at the same loading angles as for the regular square microstructure. The figure actually compares normalized tangent stiffness plots between the hierarchical and the regular square microstructure with $V^{*}=0.3$, including the corresponding failure lines. The regular square structure might be approximately twice as stiff at zero angle, compared to the hierarchical one, but its stiffness diminishes already at a small offset angle, while the stiffness of the hierarchical structure is much less affected. Even at an angle of $\pm 45^{\circ}$, the hierarchical structure has a considerable stiffness, being all in all much less anisotropic in terms of stiffness. In terms of buckling limits, the difference in anisotropy between the hierarchical and the regular square lattice is even more pronounced. When looking at the plotted failure lines, not only the radial scaling between the two graphs differs by a factor of five, but the shape of the failure lines shows a radically reduced anisotropy in terms of the compressive stability limit. Once again, it is worth noticing, that this structural design was optimized for a different loading case. 


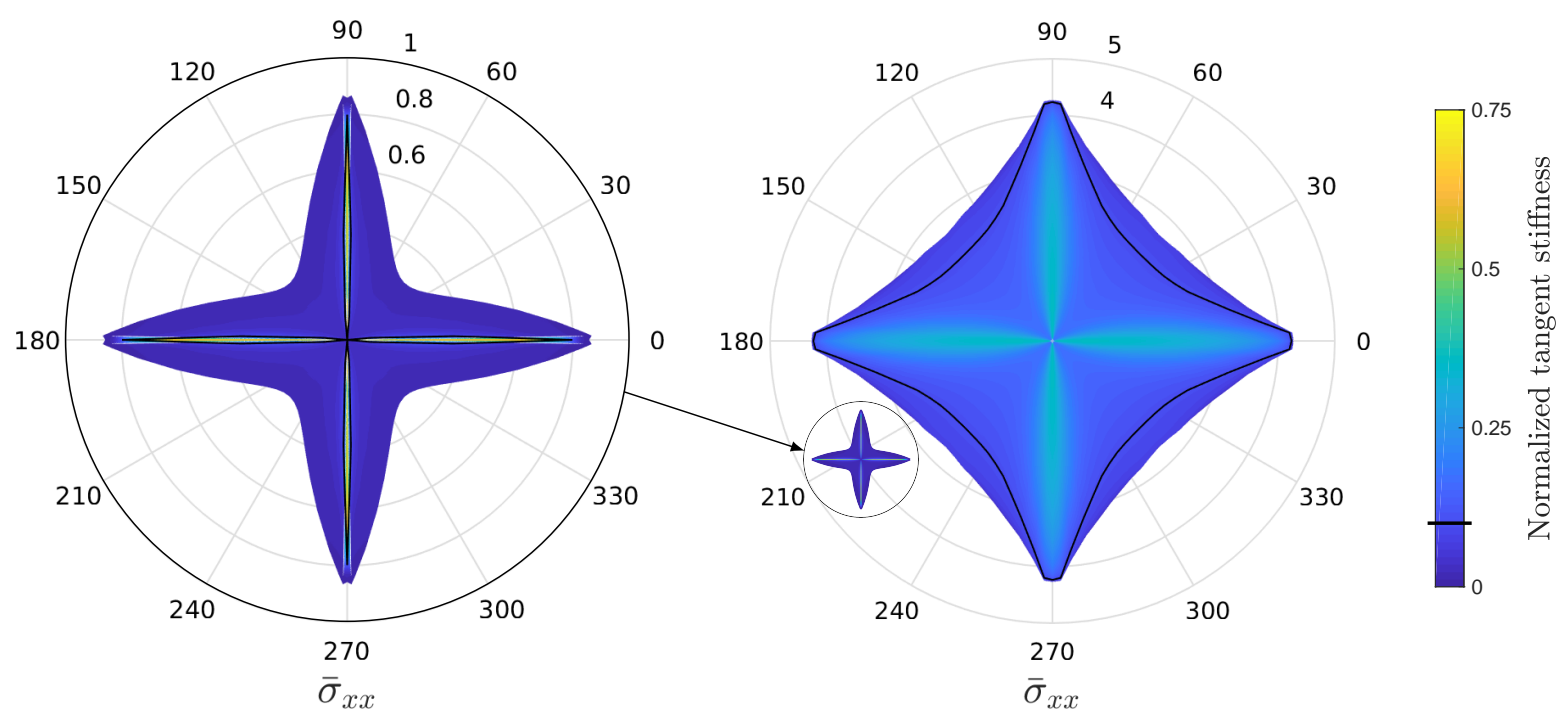

(a) Regular square

(b) Hierarchical square

Figure 16: Normalized tangent stiffness and 0.1 threshold line under rotated uniaxial loading for the regular square (a) and hierarchical (b) lattice structures of the same volume fraction. Note the different scaling of radial axis.

\subsection{Comparison of different structures at the same volume fraction}

Extending the comparison performed above to all microstructures at a volume fraction of 0.3 , this section provides a benchmarking of the considered microstructures under uniaxial loading in any direction. It is chosen to base the comparison on the two properties shown in Figure 17, namely failure lines according to section 3.2, and the initial Young's modulus in the loading direction, describing the stiffness of the structure for infinitesimal loads.

The failure lines plotted in a common diagram in Figure 17 make it clear, that the regular square microstructure has the worst buckling resistance with the dimensionless limit load not exceeding $\bar{\sigma}_{X X} \approx 0.8$ even for loading parallel to the lattice bars. At the same time it has the worst buckling resistance anisotropy compared to any of the other structures. In terms of initial stiffness, the regular square structure outperforms the other structures only within a very narrow window of load orientations, almost parallel to the lattice bars, where a relative Young's modulus of $E_{x} /\left(E V^{*}\right) \approx 0.56$ can be reached for the considered $V^{*}=0.3$.

The triangular lattice structure, which is known to be initially elastically isotropic, exhibits independent of the direction an initial stiffness of $E_{x} /\left(E V^{*}\right) \approx 0.39$, which is only $30 \%$ lower than the maximum stiffness of the regular square lattice structure. At the same time, it has the least anisotropic buckling resistance with its minimum occurring at $90^{\circ}$ and $\pm 30^{\circ}$ still being above $\bar{\sigma}_{x x} \approx 1$.

The hexagonal lattice structure is also confirmed to be initially elastically isotropic with a very low stiffness $E_{x} /\left(E V^{*}\right) \approx 0.15$, characteristic for a bending dominated structure. With regard to its buckling resistance, this structure performs similarly to the triangular one with the lowest failure limit occurring at $0^{\circ}$ and $\pm 60^{\circ}$ with a value of $\bar{\sigma}_{X X} \approx 0.77$. Of course, when comparing buckling performance, one has to keep in mind that due to the progressive softening observed, the failure line for the hexagonal structure is strongly dependent on the chosen threshold value $\eta$.

The performance of the optimized hierarchical structure in terms of stiffness and buckling resistance has already been commented upon in the previous section through a comparison to the regular square lattice structure. In addition, Figure 17 demonstrates, that in terms of buckling resistance, the hierarchical structure also outperforms all other structures considerably. Even if its buckling behavior is not as isotropic as the one of the triangular structure, its weakest direction of $\pm 45^{\circ}$ still exhibits a minimum load carrying capacity of $\bar{\sigma}_{x x} \approx 2.2$. With regard to its stiffness, the hierarchical structure is considerably less stiff than the triangular lattice structure. In its most compliant direction of $\pm 45^{\circ}$ a rather low stiffness $E_{x} /\left(E V^{*}\right) \approx 0.19$ is observed which is actually only slightly larger than the one of the hexagonal lattice structure. Apart from the hierarchical microstructure investigated here, reference [32] includes other optimized hierarchical structures that could possibly lead to even better performance. 


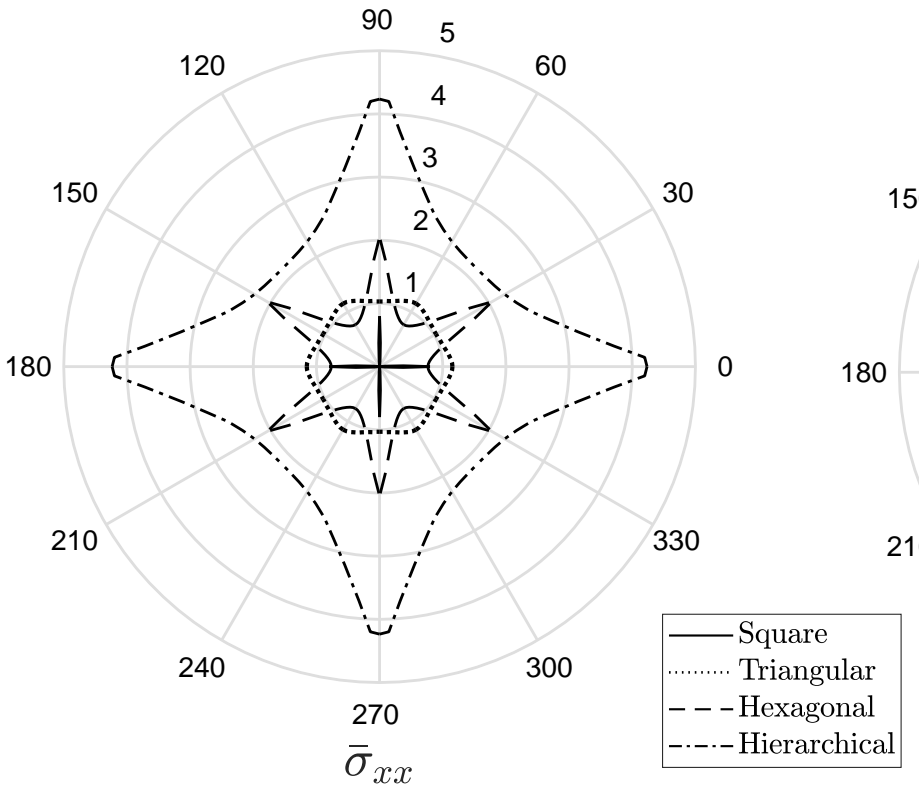

(a) Failure lines

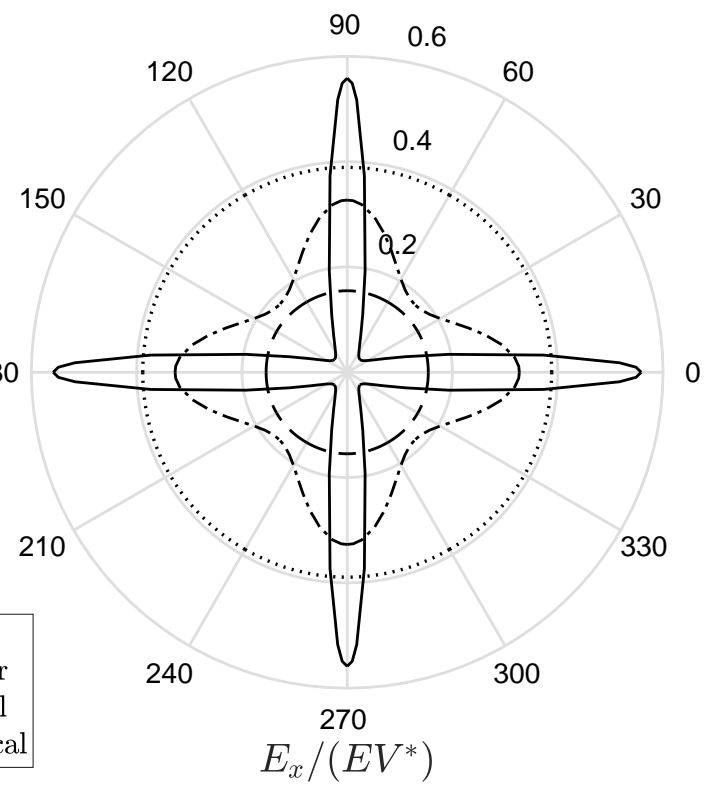

(b) Relative initial stiffness

Figure 17: Comparison of all investigated structures with $V^{*}=0.3$ under uniaxial loading. Failure lines for the regular square, triangular, hexagonal and hierarchical lattice structures (a), respectively taken from Figures $8 \mathrm{p}, 9 \mathrm{k}, 11 \mathrm{a}$ and $16 \mathrm{p}$ and relative initial stiffness for the same four lattice structures (b). $E_{x}$ is the initial modulus of elasticity for the microstructure in the load direction $x$, while $E$ is the initial Young's modulus of the solid.

\section{Conclusions}

A numerical framework has been presented for the evaluation of the compressive load carrying capacity of periodic lattice structures with finite volume fraction and arbitrarily complex geometry. It is based on a periodic cell model that includes a generalized implementation of periodic boundary conditions and homogenized strains imposed in an arbitrarily rotated frame compared to the lattice symmetry axes. Moreover, suitable dimensionless quantities have been defined for ensuring a direct comparability of microstructures with different topologies and qualitatively different responses to compressive loads. In particular, a tangential stiffness based criterion has been proposed in order to unify the treatment of structures softening progressively towards a vanishing stiffness and structures that exhibit an abrupt compressive instability. The choices of appropriate load cases and geometrical perturbations have been discussed, including a simple method proposed for generating random perturbations covering a range of wavelengths.

The presented framework has been applied for the evaluation of the three most common simple lattices in 2D, i.e. square, triangular and hexagonal, at three volume fractions, as well as for studying an optimized hierarchical microstructure from the literature. The included results enhance the state-of-the-art with stability limits reported for the three classical lattices considered, demonstrating the strong effect of finite volume fractions compared to the known limits at infinitesimal volume fractions. The choice of analyzing structures of a given volume fraction instead of a given bar slenderness ratio allowed the direct comparison of lattices in terms of material utilization. The final comparison between the performance of all four considered microstructures at a volume fraction of 0.3 , in terms of both compressive stability and initial stiffness, provides a very useful overview. At the same time it has verified the much superior buckling resistance of the optimized hierarchical structure, despite its relatively low directional stiffness. Here we remind, that [32] also suggests hierarchical hexagonal and triangular structures.

The hierarchical microstructure treated in this work, has also been used as an example for demonstrating the complexity of the nonlinear response of a generic microstructure when finite deformations are accounted for. Plots of the deformed structure at overall strains in order of $20 \%$ just before buckling have illustrated how severely distorted the geometry can be in the pre-buckled state. Moreover, cases of switching between two buckling modes in the post-buckling region have provided additional insight in the post-buckling behavior of this structure. 


\section{Acknowledgements}

This work was supported by the Villum Fonden through the Villum investigator project InnoTop.

\section{References}

[1] L. J. Gibson and M. F. Ashby. Cellular Solids: Structure and Properties. Cambridge Solid State Science Series. Cambridge University Press, 2 edition, 1997.

[2] D. Mahmoud and M. A. Elbestawi. Lattice structures and functionally graded materials applications in additive manufacturing of orthopedic implants: A review. Journal of Manufacturing and Materials Processing, 2017.

[3] A. Vyatskikh, S. Delalande, A. Kudo, X. Zhang, C. M. Portela, and J. R. Greer. Additive manufacturing of 3d nano-architected metals. Nature Communications, 9(1):593, 2018.

[4] M. Helou and S. Kara. Design, analysis and manufacturing of lattice structures: an overview. International Journal of Computer Integrated Manufacturing, 31(3):243-261, 2018.

[5] T. C. Triantafillou, J. Zhang, T. L. Shercliff, L. J. Gibson, and L. J. Ashby. Failure surfaces for cellular materials under multiaxial loads-II. Comparison of models with experiment. International Journal of Mechanical Sciences, 31(9):665 - 678, 1989.

[6] S. D. Papka and S. Kyriakides. In-plane compressive response and crushing of honeycomb. Journal of the Mechanics and Physics of Solids, 42(10):1499-532, 1499-1532, 1994

[7] S. D. Papka and S. Kyriakides. Biaxial crushing of honeycombs - Part I: Experiments. International Journal of Solids and Structures, 36(29):4367-4396, 1999

[8] H. X. Zhu and N. J. Mills. The in-plane non-linear compression of regular honeycombs. International Journal of Solids and Structures, 37(13):1931-1949, 2000.

[9] M. P. Bendsøe and O. Sigmund. Material interpolation schemes in topology optimization. Archive of Applied Mechanics, 69(9):635-654, Nov 1999.

[10] A. J. Wang and D. L. McDowell. In-plane stiffness and yield strength of periodic metal honeycombs. Journal of Engineering Materials and Technology, Transactions of the Asme, 126(2):137-156, 2004.

[11] L. R. Meza, G. P. Phlipot, C. M. Portela, A. Maggi, L. C. Montemayor, A. Comella, D. M. Kochmann, and J. R. Greer. Reexamining the mechanical property space of three-dimensional lattice architectures. Acta Materialia, 140:424-432, 2017.

[12] I. Maskery, A.O. Aremu, L. Parry, R.D. Wildman, C.J. Tuck, and I.A. Ashcroft. Effective design and simulation of surface-based lattice structures featuring volume fraction and cell type grading. Materials $\mathcal{E}$ Design, 155:220 - 232, 2018.

[13] L. J. Gibson, M. F. Ashby, J. Zhang, and T. C. Triantafillou. Failure surfaces for cellular materials under multiaxial loads-I.Modelling. International Journal of Mechanical Sciences, 31(9):635 - 663, 1989.

[14] N. Triantafyllidis and M. W. Schraad. Onset of failure in aluminum honeycombs under general in-plane loading. Journal of the Mechanics and Physics of Solids, 46(6):1089-1124, 1998.

[15] N. Ohno, D. Okumura, and H. Noguchi. Microscopic symmetric bifurcation condition of cellular solids based on a homogenization theory of finite deformation. Journal of the Mechanics and Physics of Solids, 50(5):1125-1153, 2002.

[16] A. J. Wang and D. L. McDowell. Yield surfaces of various periodic metal honeycombs at intermediate relative density. International Journal of Plasticity, 21(2):285-320, 2005.

[17] H. C. Tankasala, V. S. Deshpande, and N. A. Fleck. Tensile response of elastoplastic lattices at finite strain. Journal of the Mechanics and Physics of Solids, 109:307 - 330, 2017.

[18] J. C. Michel, O. Lopez-Pamies, P. Ponte Castañeda, and N. Triantafyllidis. Microscopic and macroscopic instabilities in finitely strained porous elastomers. Journal of the Mechanics and Physics of Solids, 55(5):900 - 938, 2007.

[19] V. S. Deshpande, N. A. Fleck, and M. F. Ashby. Effective properties of the octet-truss lattice material. Journal of the Mechanics and Physics of Solids, 49(8):1747 - 1769, 2001.

[20] S. Xu, J. Shen, S. Zhou, X. Huang, and Y. M. Xie. Design of lattice structures with controlled anisotropy. Materials and Design, 93:443-447, 2016.

[21] T. Tancogne-Dejean and D. Mohr. Elastically-isotropic truss lattice materials of reduced plastic anisotropy. International Journal of Solids and Structures, 138:24 - 39, 2018

[22] J. S. Huang and L. J. Gibson. Fracture toughness of brittle honeycombs. Acta Metallurgica Et Materialia, 39(7):1617-1626, 1991.

[23] J. Y. Chen, Y. Huang, and M. Ortiz. Fracture analysis of cellular materials: A strain gradient model. Journal of the Mechanics and Physics of Solids, 46(5):789-828, 1998.

[24] N. A. Fleck and X. Qiu. The damage tolerance of elastic-brittle, two-dimensional isotropic lattices. Journal of the Mechanics and Physics of Solids, 55(3):562-588, 2007.

[25] F. Lipperman, M. Ryvkin, and M. B. Fuchs. Fracture toughness of two-dimensional cellular material with periodic microstructure. International Journal of Fracture, 146(4):279-290, 2007.

[26] S. P. Timoshenko and J. G. Gere. Theory of elastic stability. McGraw-Hill, 1961.

[27] S. D. Papka and S. Kyriakides. In-plane biaxial crushing of honeycombs - : Part II: Analysis. International Journal of Solids and Structures, 36(29):4397-4423, 1999.

[28] B. Haghpanah, J. Papadopoulos, D. Mousanezhad, H. Nayeb-Hashemi, and A. Vaziri. Buckling of regular, chiral and hierarchical honeycombs under a general macroscopic stress state. Proceedings of the Royal Society A: Mathematical, Physical and Engineering Sciences, 470(2167):20130856, 2014

[29] R. De Pascalis, M. Destrade, and A. Goriely. Nonlinear correction to the euler buckling formula for compressed cylinders with guided-guided end conditions. Journal of Elasticity, 102(2):191-200, Feb 2011. 
[30] X. Li, Z. Lu, Z. Yang, and C. Yang. Anisotropic in-plane mechanical behavior of square honeycombs under off-axis loading. Materials and Design, 158:88-97, 2018

[31] X. Zheng, H. Lee, T. H. Weisgraber, M. Shusteff, J. DeOtte, E. B. Duoss, J. D. Kuntz, M. M. Biener, Q. Ge, J. A. Jackson, S. O. Kucheyev, N. X. Fang, and C. M. Spadaccini. Ultralight, ultrastiff mechanical metamaterials. Science, 344(6190):1373-1377, 2014.

[32] C. R. Thomsen, F. Wang, and O. Sigmund. Buckling strength topology optimization of $2 \mathrm{~d}$ periodic materials based on linearized bifurcation analysis. Computer Methods in Applied Mechanics and Engineering, 339:115-136, 2018.

[33] T. Frenzel, C. Findeisen, M. Kadic, P. Gumbsch, and M. Wegener. Tailored buckling microlattices as reusable light-weight shock absorbers. Advanced Materials, 28(28):5865-5870, 2016.

[34] D. M. Kochmann and K. Bertoldi. Exploiting microstructural instabilities in solids and structures: From metamaterials to structural transitions. Applied Mechanics Reviews, 69(5):050801, 2017.

[35] J.D. Clayton. Nonlinear Mechanics of Crystals. Solid Mechanics and Its Applications. Springer Netherlands, 2011.

[36] U. F. Kocks. The relation between polycrystal deformation and single-crystal deformation. Metallurgical and Materials Transactions, 1(5):1121-1143, 1970.

[37] J. C. Simo, R. L. Taylor, and K. S. Pister. Variational and projection methods for the volume constraint in finite deformation elasto-plasticity. Computer Methods in Applied Mechanics and Engineering, 51(1-3):177-208, 1985.

[38] GetFEM - An open-source finite element library. http://getfem.org

[39] K. Poulios and C. F. Niordson. Micro-buckling of periodically layered composites in regions of stress concentration. Composite Structures, $157: 424-435,2016$

[40] F. Wang and O. Sigmund. Numerical investigation of stiffness and buckling response of simple and optimized infill structures (accepted). Structural and Multidisciplinary Optimization, 2019

[41] O. Rokoš, M.M. Ameen, R.H.J. Peerlings, and M.G.D. Geers. Micromorphic computational homogenization for mechanical metamaterials with patterning fluctuation fields. Journal of the Mechanics and Physics of Solids, 123:119 - 137, 2019. The N.A. Fleck 60th Anniversary Volume.

[42] K. Bertoldi, M. C. Boyce, S. Deschanel, S. M. Prange, and T. Mullin. Mechanics of deformation-triggered pattern transformations and superelastic behavior in periodic elastomeric structures. Journal of the Mechanics and Physics of Solids, 56(8):2642-2668, 2008

[43] N. Triantafyllidis and W. C. Schnaidt. Comparison of microscopic and macroscopic instabilities in a class of two-dimensional periodic composites. Journal of the Mechanics and Physics of Solids, 41(9):1533-65, 1533-1565, 1993 